\title{
Review Article \\ Recent Progress in Triboelectric Nanogenerators as a Renewable and Sustainable Power Source
}

\author{
Zhiming Lin, ${ }^{1}$ Jun Chen, ${ }^{2}$ and Jin Yang ${ }^{1}$ \\ ${ }^{1}$ Department of Optoelectronic Engineering, Chongqing University, Chongqing 400044, China \\ ${ }^{2}$ School of Materials Science and Engineering, Georgia Institute of Technology, Atlanta, GA 30332, USA
}

Correspondence should be addressed to Jin Yang; yangjin@cqu.edu.cn

Received 19 August 2015; Accepted 20 January 2016

Academic Editor: David Cornu

Copyright (C) 2016 Zhiming Lin et al. This is an open access article distributed under the Creative Commons Attribution License, which permits unrestricted use, distribution, and reproduction in any medium, provided the original work is properly cited.

The newly developed triboelectric nanogenerators (TENGs) provide an excellent approach to convert mechanical energy into electricity, which are mainly based on the coupling between triboelectrification and electrostatic induction. The TENG has the potential of harvesting many kinds of mechanical energies such as vibration, rotation, wind, human motion, and even water wave energy, which could be a new paradigm for scavenging large scale energy. It also demonstrates a possible route towards practical applications for powering electronic devices. This paper presents a comprehensive review of the four modes of TENGs: vertical contact-separation mode, in-plane sliding mode, single-electrode mode, and free-standing triboelectric-layer mode. The performance enhancements of TENGs for harvesting energy as a sustainable power source are also discussed. In addition, recent reports on the hybridized nanogenerator are introduced, which may enable fully self-powered electronic devices. Finally, the practical applications of TENGs for energy harvesting are presented.

\section{Introduction}

Energy is one of the key factors which influences the quality of our life and sustainable development of modern society. In dealing with the increased energy consumption from limited traditional fossil fuel sources on the earth, searching for sustainable power sources with reduced carbon emissions and studying renewable energy technologies are urgent for the sustainable development of human civilization. The familiar renewable energies such as wind and solar and thermal energy are targeted to meet the need of megawatt to gigawatt power scales as power sources. The requirements for harvesting these energies are high power density, high efficiency, and low cost.

Over the past decades, everything that hallmarks the high-tech era, from handheld cell phones to portable and even wearable electronic devices, depends on electricity, which has become indispensable in people's daily life. Moreover, the tremendous sensors for health monitoring, personal medical networks, military surveillance, environmental/infrastructure monitoring, and security have been applied and distributed in every corner of our life. And the massive development of electronic devices is towards miniaturization, light weight, and portability. They then require power solutions that are sustainable, available, maintenance-free, and even perpetual for every unit. Generally, the electronic devices use batteries as the external power sources. Due to the limited lifetime, the environmental pollution problem, the replacement of batteries and the large number of devices, and vast scope of distribution, it is thus desirable to replace them by harvesting energy from the ambient environment as sustainable self-sufficient power sources to maintain independent and continuous operations of these electronics. In this regard, energy harvesting techniques have been developed for supplying energy to electronic devices by converting ambient energy into electrical energy. Mechanical energy, due to its abundance, has been one of the major energy sources for energy harvesting systems [1-4]. To date, many mechanisms of energy harvesting techniques have been developed that are based on various mechanisms including piezoelectric effect, electrostatic effect, and electromagnetic induction, which have been extensively developed for a few decades [5-11].

Most recently, a new type of energy harvesting technology named as triboelectric generator (TENG) has been invented 


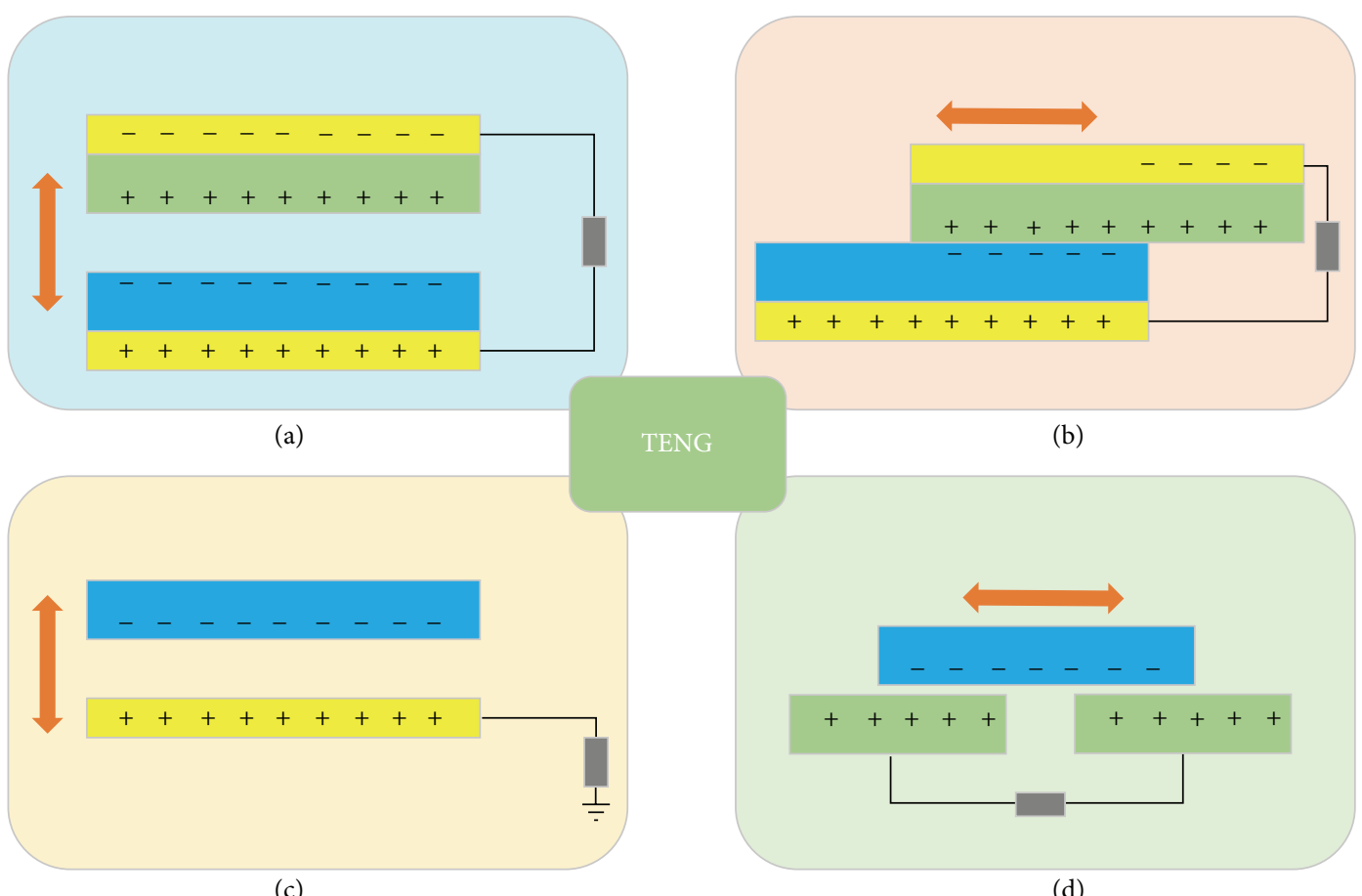

(c)

(d)

Figure 1: The four fundamental modes of the TENG. (a) The vertical contact-separation mode. (b) The in-plane sliding mode. (c) The singleelectrode mode. (d) The free-standing triboelectric-layer mode.

as an alternative method for scavenging the ambient mechanical energy in the environment to electricity [12-16]. The TENG has a novel and unique mechanism which operates by a conjunction of triboelectrification and electrostatic induction through the contact-separation or relative sliding between two materials that have opposite tribopolarity. After the introduction of TENG in 2012, it has attracted increasing interest for converting mechanical energy into electricity and for meeting large scale energy demands. Fundamentals that rely on the coupling between the triboelectric effect and electrostatic induction have been reported, and various device structures which can harness all kinds of mechanical energies such as vibration [17-20], human motion [21, 22], rotation $[23,24]$, wind $[25,26]$, flowing water [27], and walking [28] have been demonstrated. A variety of applications using this technology for energy harvesting or sensing purposes have been represented [29-34]. According to the existing reviews on TENGs, this paper covers the recent progress in TENGs as a renewable and sustainable power source.

\section{Fundamentals}

The triboelectric effect is a well-known phenomenon that refers to the charge generation between two different materials with distinct surface electron affinities. When they are brought into contact through friction, the different potential is created by the separation of the two material surfaces. Electrostatic induction phenomenon is an electricity-generating process such that electrons in one electrode would flow to the other electrode through the external load in order to balance the potential difference. As for TENGs, they realize the conversion of mechanical energy into usable electricity by the integration of triboelectrification with electrostatic inductions $[35,36]$. Four fundamental modes of the TENG including vertical contact-separation mode [37-40], in-plane sliding mode [41, 42], single-electrode mode [43-46], and free-standing triboelectric-layer mode [47-52] have been proposed and demonstrated, as shown in Figure 1.

2.1. Vertical Contact-Separation Mode. The working principle of TENGs for the case of vertical contact-separation mode can be depicted by the coupling between contact charging and electrostatic induction. Zhu et al. are the first to report an accurate and systematic description of the triboelectrification-driven energy conversion process in January 2012 [13]. A full cycle of the electricity generation process of vertical contact-separation mode TENG is illustrated in Figure 2. Polymethyl methacrylate (PMMA) and polyimide (Kapton) are employed as the two contact materials.

Under open-circuit conditions, there is no charge generated or induced; therefore, no electric potential difference (EPD) between the two electrodes emerges (Figure 2(a)(I)). When two dielectric materials are applied by an external force, the two materials are brought into contact with each other. Surface charge transfer then occurs on these two contacting surfaces due to triboelectric effect (Figure 2(a)(II)). As determined by the triboelectric series, electrons are injected from the surface of PMMA into that of Kapton, resulting in the accumulation of net positive charges on the PMMA side and net negative charges on the Kapton side. Once there is a relative separation between two materials due to the resilience, EPD is then established between the two electrodes 


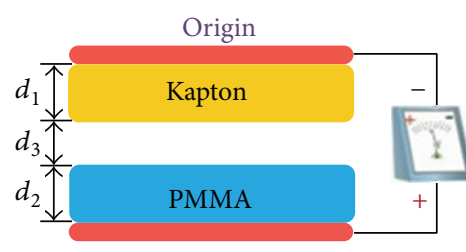

(I)

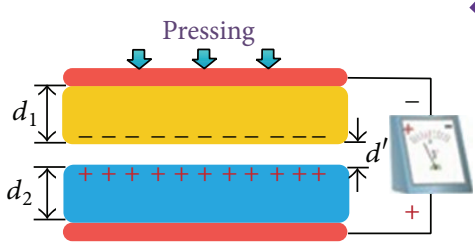

(VI)

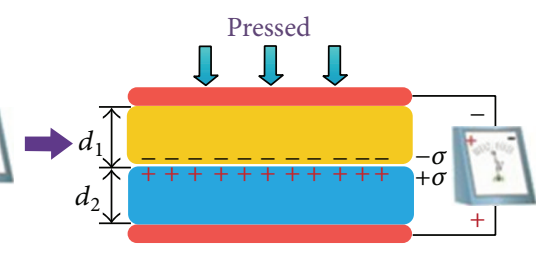

(II)

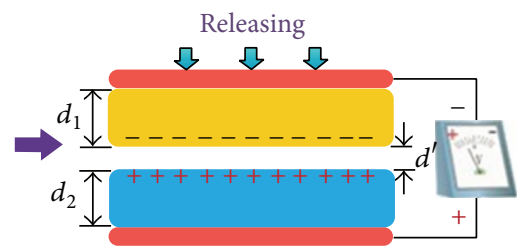

(III)

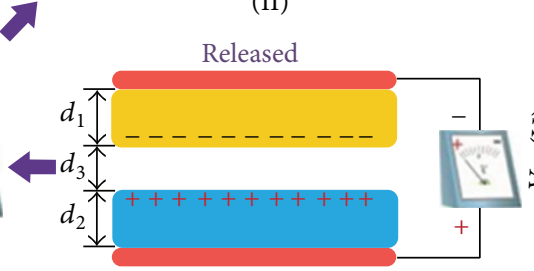

(V)

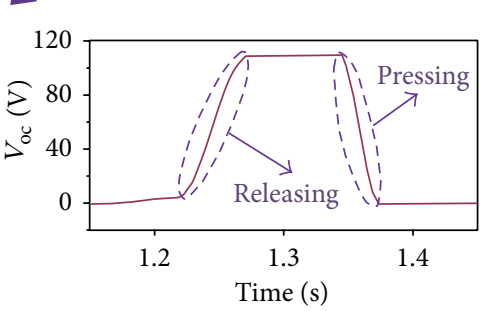

(IV)

(a)

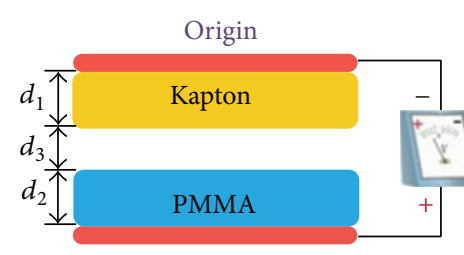

(I)

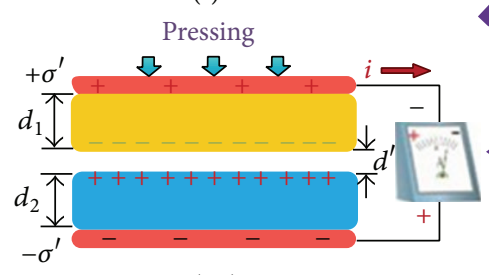

(VI)

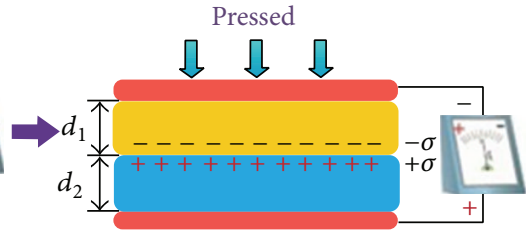

(II)
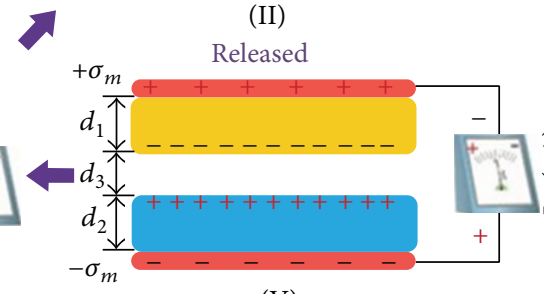

(V)

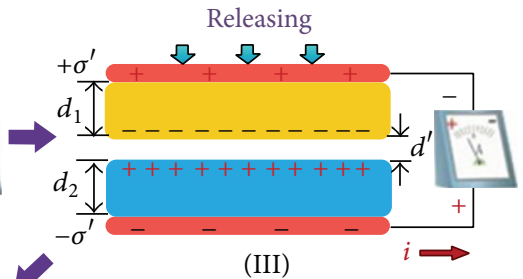

(III)

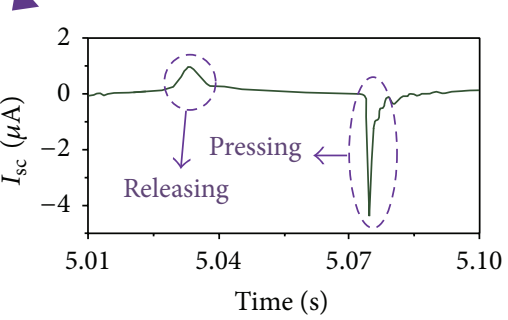

(IV)

(b)

FIGURE 2: Electricity generation process in a full cycle of the TENG for vertical contact-separation mode [13]. (a) Open-circuit. (b) Shortcircuit.

(Figure 2(a)(III)). In this case, EPD (equivalent to opencircuit voltage) between the two electrodes will be induced. If we define electric potential of the bottom electrode to be zero, it can be expressed as

$$
V_{\text {oc }}=-\frac{\sigma d}{\varepsilon_{0}},
$$

where $\sigma$ is the triboelectric charge density, $\varepsilon_{0}$ is the vacuum permittivity, and $d$ is the gap between the two contact materials. As the Kapton film is being released, the opencircuit voltage increases and reaches the maximum value when the Kapton film fully moves backward to the original state (Figure $2(\mathrm{a})(\mathrm{V})$ ). If the external force is applied again, the electric potential difference begins to diminish when the two materials get closer to each other. As a result, $V_{\text {oc }}$ descends from the maximum value to zero when a full contact is made again (Figure 2(a)(VI), (II)). This is a full cycle of the electricity-generating process.
Under short-circuit conditions, any electric potential difference as the Kapton film moves upward drives electrons to flow from the top electrode to the bottom electrode (Figure 2(b)(III)) in order to balance the generated triboelectric potential, resulting in an instantaneous positive current in the releasing process (Figure 2(b)(IV)). The net effect is that the induced charges accumulate with positive sign on the top electrode and negative sign on the bottom electrode (Figure $2(\mathrm{~b})(\mathrm{V}))$. The induced charge density $\left(\sigma^{\prime}\right)$ when the Kapton film is fully released can be expressed as

$$
\sigma^{\prime}=\frac{\sigma d^{\prime} \varepsilon_{r k} \varepsilon_{r p}}{d_{1} \varepsilon_{r p}+d^{\prime} \varepsilon_{r k} \varepsilon_{r p}+d_{2} \varepsilon_{r k}}
$$

where $\varepsilon_{r k}$ and $\varepsilon_{r p}$ are the relative permittivity of Kapton and PMMA, respectively, and $d_{1}$ and $d_{2}$ are the thickness of the Kapton film and the PMMA layer, respectively. The maximum value of $\sigma_{\max }^{\prime}$ can be obtained by substituting $d_{3}$, the 


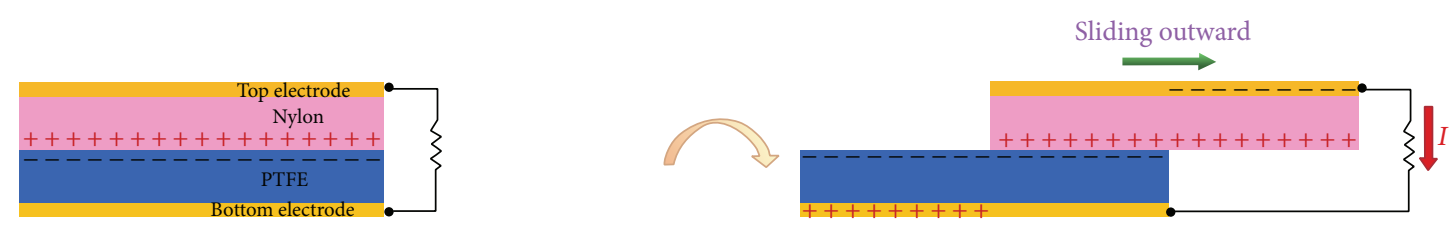

(I)

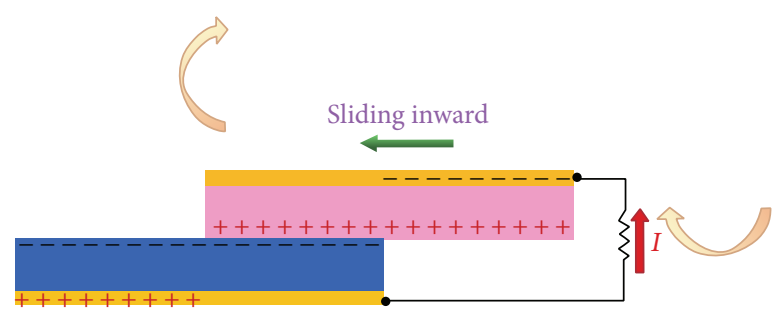

(IV)

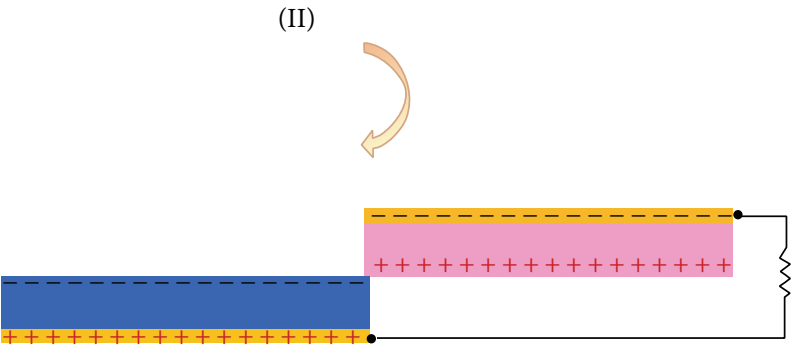

(III)

(a)

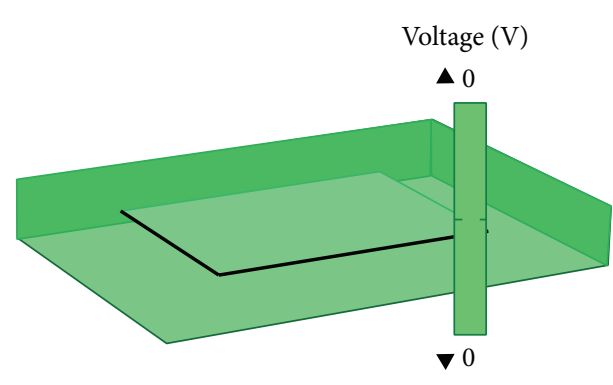

(b)

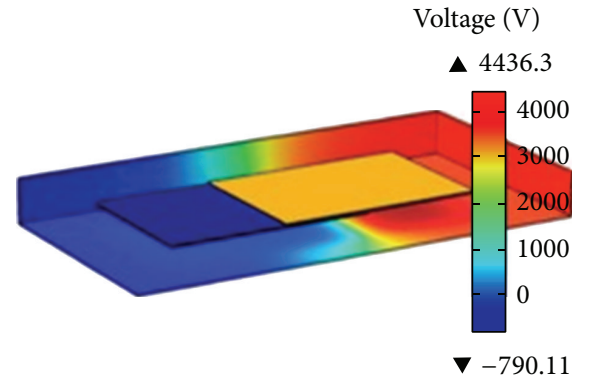

(c)

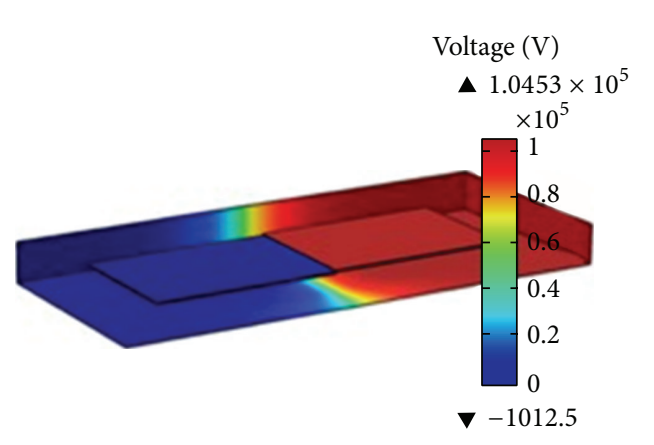

(d)

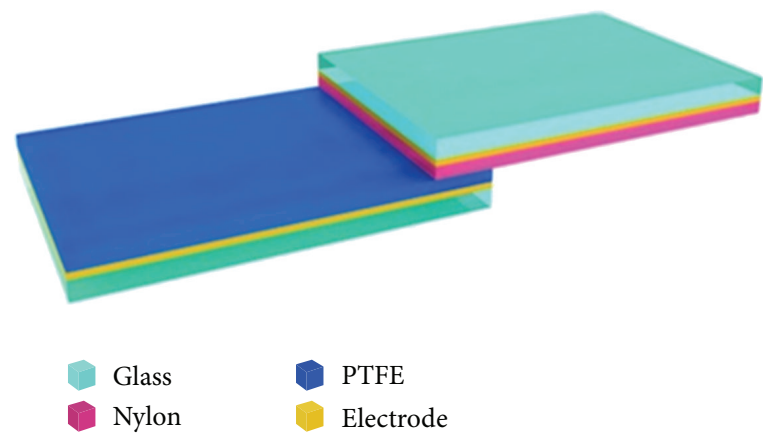

(e)

FIGURE 3: Operating principle of the in-plane sliding mode TENG [42]. (a) Schematic illustrations showing the electricity generation within a full cycle of the in-plane sliding mode. (b-d) Simulation result of the potential difference between the two electrodes at different sliding positions: (b) the overlapping position; (c) sliding halfway out; and (d) fully sliding out. (e) Schematic illustrations of the in-plane sliding mode TENG.

gap height for $d^{\prime}$ in the equation above. Once the generator is applied by an external force again, the distance of the two interlayers would reduce, leading to the top electrode possessing a higher electric potential than the bottom electrode. As a result, electrons are driven from the bottom electrode back to the top electrode, reducing the amount of induced charges (Figure 2(b)(VI)). This process corresponds to an instantaneous negative current (Figure 2(b)(IV)). As the two interlayers are in full contact again, induced charges are all neutralized (Figure 2(b)(II)). Because of reciprocating motion of the materials, the generator can produce alternating current.
2.2. In-Plane Sliding Mode. In 2013, Wang et al. demonstrated a new type of TENG that is designed based on the in-plane sliding between the two surfaces in lateral direction [42]. The TENG consists of a triboelectric PTFE patch, a Nylon plate, and two electrodes. The PTFE patch and the Nylon plate are arranged in parallel to each other, where the inner surfaces are in intimate contact (Figure 3(e)).

The sliding-induced electricity generation mechanism is schematically depicted in Figure 3(a). At the original position, the surfaces of Nylon and PTFE are in full contact with each other. Because of the distinct difference in the ability to attract electrons, the contact between the Nylon and 
PTFE will result in electrons injected from Nylon to PTFE. During this period, there is no potential difference between the two electrodes (Figure 3(a)(I)). Once the top plate with the positively charged surface starts to slide outward (Figure 3(a)(II)), relative displacement in contact mode occurs in lateral direction. There will be a higher potential on PTFE's electrode than on Nylon's electrode; thus the electrons in the electrode attached to the PTFE film will be driven to Nylon's electrode through the external load. After that the potential difference and the amount of transferred charges reach the maximum values until the two plates reach full mismatch (Figure 3(a)(III)). When the top Nylon plate is reverted to sliding inward (Figure 3(a)(IV)), the transferred charges on the electrodes will flow back through the external load and produce a negative current signal to keep the electrostatic equilibrium. When the two plates completely reach the original position, there will be no transferred charges left on the electrode. Thus no output current can be observed. Figures 3(b)-3(d) show the in-plane charge-separation-induced electric potential distribution and charge transfer through numerical simulation using COMSOL. As shown by the simulation results, the potential difference keeps increasing with the increase of the displacement.

There are several very important advantages of the inplane sliding mode comparing with the vertical contact-separation mode. Energy conversion efficiency is improved owing to the full contact. Furthermore, the more advanced design for high-performance TENGs is developed easily based on the in-plane sliding mode. For instance, through a multilayered disk structure, the total amount of transported charges is greatly enhanced [53]. The effective power enhancement can be achieved.

2.3. Single-Electrode Mode. The TENGs presented in the above sections must have two electrodes to form a closed circuit for the electrons to flow. However, such device configuration largely limits the practical applications of harvesting energy from an arbitrary, freely moving object. In this regard, the single-electrode mode TENG was demonstrated to solve this problem [45]. Figure 4(a) shows a schematic diagram of the single-electrode mode TENG. A PDMS film which is uniformly covered with an array of micropyramids serves as the friction surface. And the surface of skin is employed as the other contact surface. A transparent ITO induction electrode is coated on the back side of the PET substrate. The change of distance between two surfaces results in charge transfer between the ITO electrode and the ground, thus driving the flow of electrons through an external load.

The energy harvesting mechanism of the TENG is schematically shown in Figure 4(b). When a human finger is brought into contact with PDMS, the charge transfer between them at the contact interface occurs (Figure 4(b)(I)). Since PDMS is much more triboelectrically negative than human skin, it is generating positive triboelectric charges on the human skin and negative ones on the FEP. Electrons are injected from human skin into PDMS. The produced negative triboelectric charges on the PDMS surface can be preserved for a long time due to the nature of the insulator. As the human finger separates from the PDMS surface, a potential difference is generated between the ITO electrode and the grounded reference electrode. The negative charges on the PDMS side will induce positive charges on the ITO electrode, resulting in a flow of free electrons via the external load from the ITO electrode to ground in order to reach an electrostatic equilibrium state, as depicted in Figure 4(b)(II). When the human finger is reverted to approaching the PDMS again, the free electrons flow backward from the ground to the ITO electrode until the skin and PDMS film are in full contact with each other again resulting in producing a negative voltage/ current signal, as shown in Figure 4(b)(IV). This is a full cycle of the single-electrode-based sliding TENG working process.

2.4. Free-Standing Triboelectric-Layer Mode. The free-standing triboelectric-layer mode TENG has the advantages of versatility and applicability for harvesting energy from an arbitrary moving object or a walking human without an attached electrode. And the free-standing triboelectric-layer mode features ultrarobustness as well as high energy conversion efficiency [47]. The operation of the TENG based electricity generation relies on relative position change of the tribocharged surface between two electrodes resulting in a periodic change of the induced potential difference, as shown in Figure 5(a). The structure of the TENG is composed of a free-standing dielectric layer and two metal films. The two metal films play dual roles of the triboelectric material and two electrodes. The electricity-generating process is elaborated through a basic unit in Figure 5(b).

In the initial state, when the FEP layer is aligned with the left-hand electrode in direct contact, due to their different abilities in attracting electrons, there will be net negative charges on the inner surface of the FEP layer and net positive charges on the surface of the left-hand electrode (Figure 5(b)(I)). Then, when the FEP layer slides towards the right-hand electrode (Figure 5(b)(II)), the EDP between the right-hand electrode and the left-hand electrode will be gradually reduced, thus resulting in a current flow from the left-hand electrode to the right-hand electrode via the external load in order to reduce the potential difference (Figure 5(b)(II)). Once the FEP plate completely reaches the overlapping position of the right-hand electrode, no current flows through the external load (Figure 5(b)(III)). When the FEP plate is reverted to sliding backward, an alternating current is produced in the external load (Figure 5(b)(IV)). This is the cycle of electricity generation process. Besides, the other basic design of free-standing triboelectric-layer mode TENGs is based on the triboelectrification such as swinging the FEP between the two electrodes even without direct contact (Figure 5(c)) and two different dielectric films' structure (Figure 5(d)).

\section{High-Performance Triboelectric Nanogenerators as a Sustainable Power Source}

The triboelectric nanogenerator offers a completely innovative approach for energy harvesting from the vast environment due to its high power density, light weight, small size, 

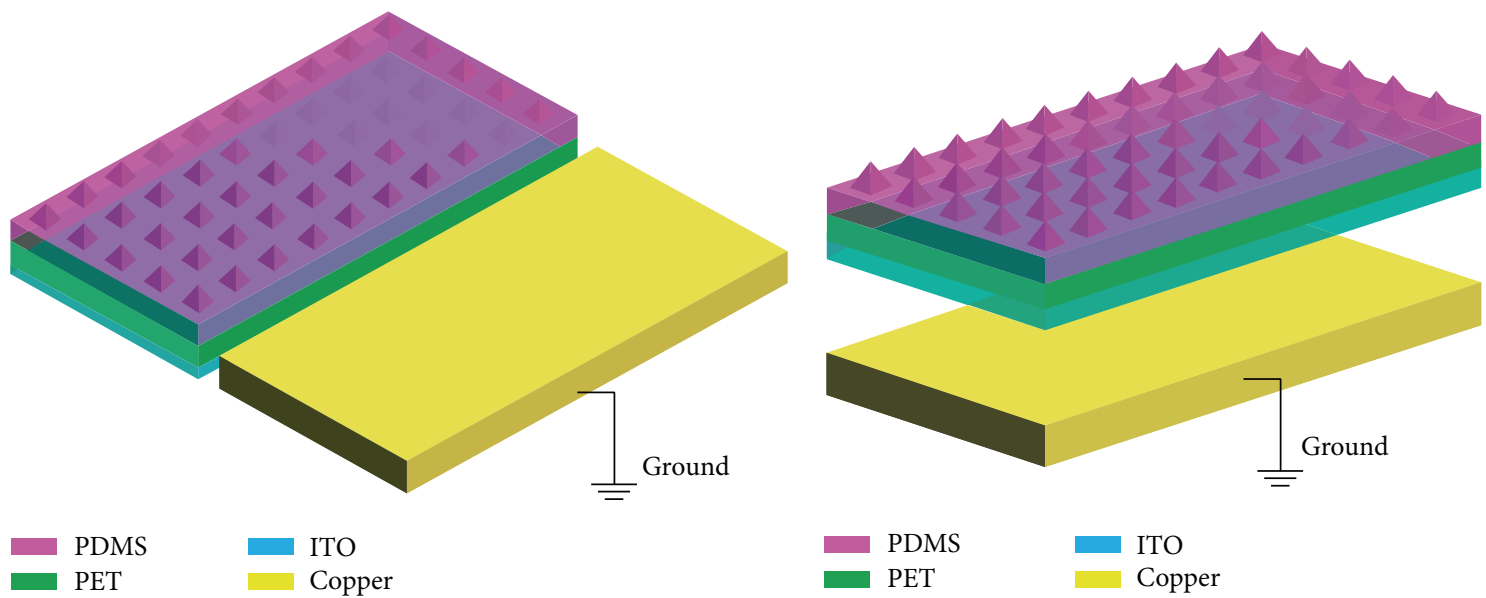

(II)

(a)

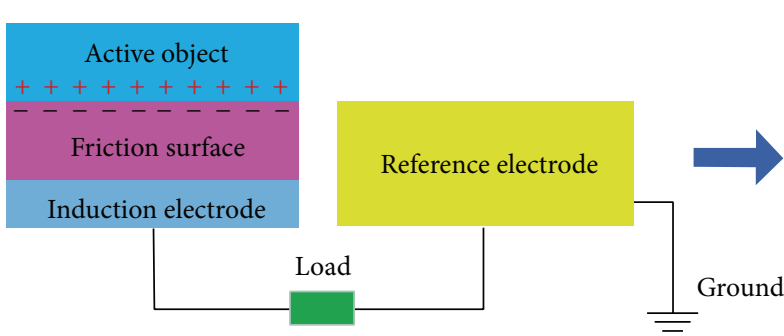

(I)
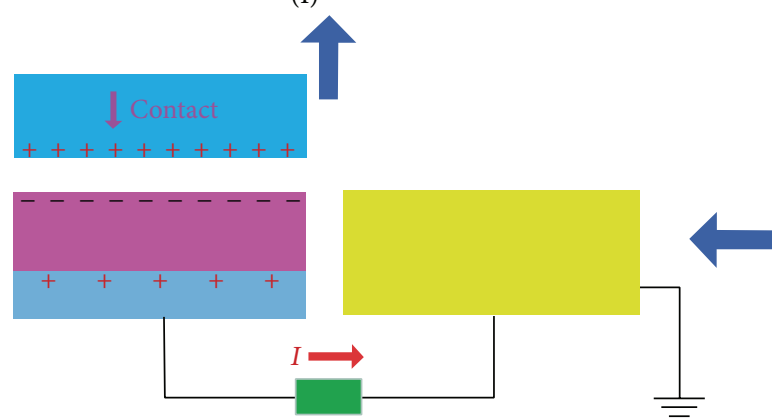

(IV)

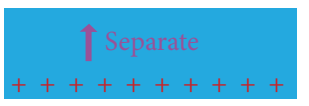

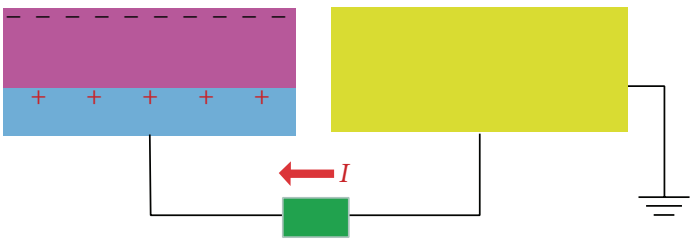

(II)
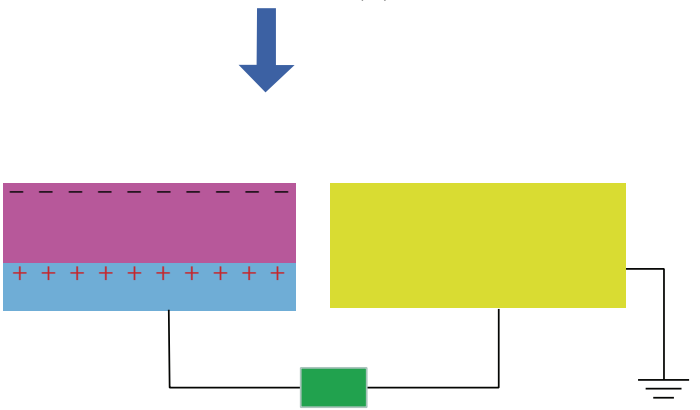

(III)

(b)

FIGURE 4: (a) Schematic illustration showing the single-electrode mode TENG [45]. (b) Sketches that illustrate the cycle of the electricity generation process.

and so on. However, several output performances of the TENG should be improved such as output power, current, and energy conversion efficiency. Hence, many principles and mechanisms have been demonstrated to realize the enhancement of TENGs as a sustainable power source for electronics $[14,53-62]$.

3.1. Enhancing Electric Output for Large Scale Energy Harvesting. In order to obtain higher power output to meet large needs of energy, a large scale energy harvester by a nanoparticle-enhanced triboelectric nanogenerator has been developed [14]. Fabrication of the TENG is relatively simple, with easy processing and low cost. The structure of the TENG consists of two substrates that are connected by four springs to maintain a gap, as shown in Figure 6(a). PMMA is employed as the material for substrates. On the bottom side, a layer of contact electrode is prepared. The contact electrode which is a gold thin film with uniformly sized and distributed nanoparticles plays dual roles of electrode and contact surface (Figure 6(b)). The nanoparticles-based modification will further increase the effective contact area, thus enhancing the electrical output of TENGs. On the top side, a thin film of gold which is employed as another electrode is sandwiched by a layer of polydimethylsiloxane (PDMS) and the substrate. The as-fabricated TENG is presented in Figure 6(c). 

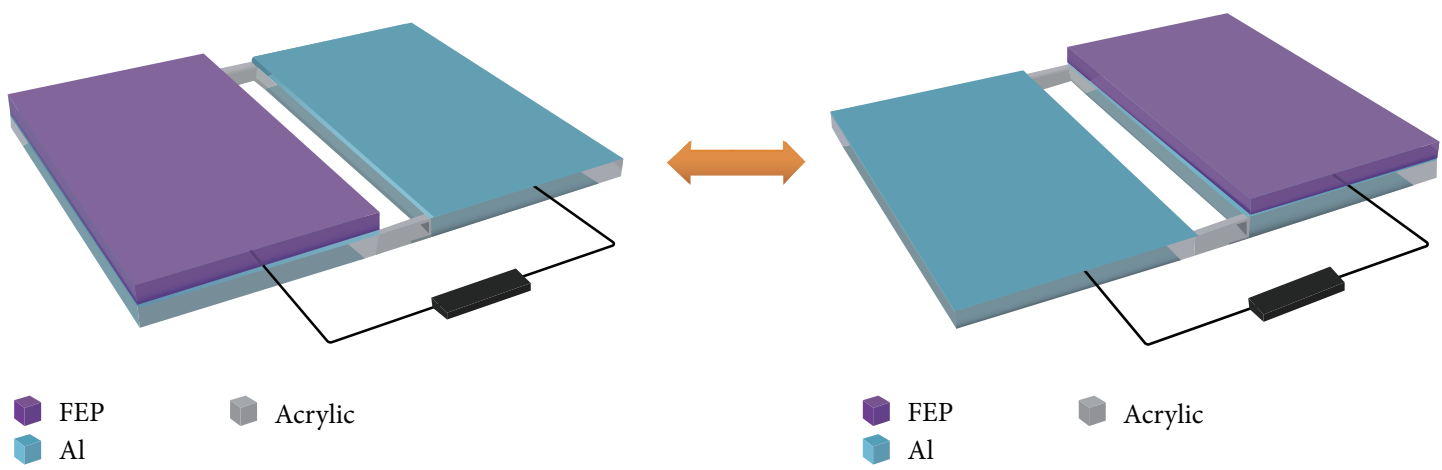

(a)

Dielectric-to-conductor FTENG in contact-sliding mode

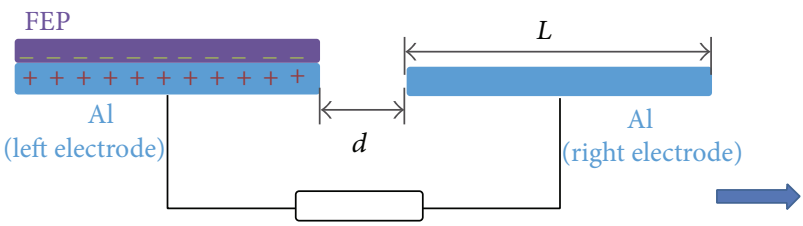

(I)

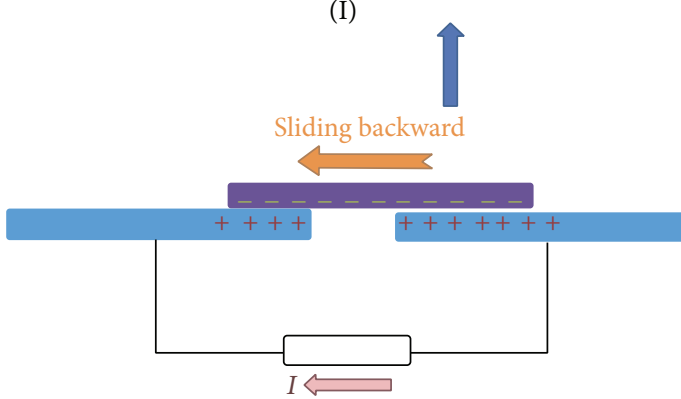

(IV)

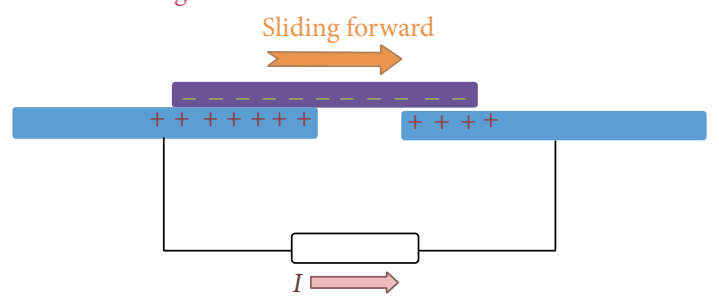

(II)
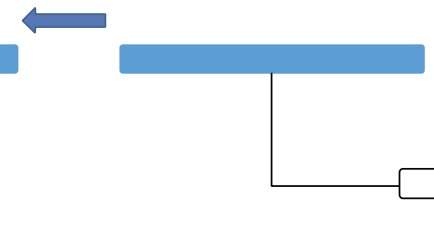

(b)

Dielectric-to-conductor FTENG in non-contact-sliding mode

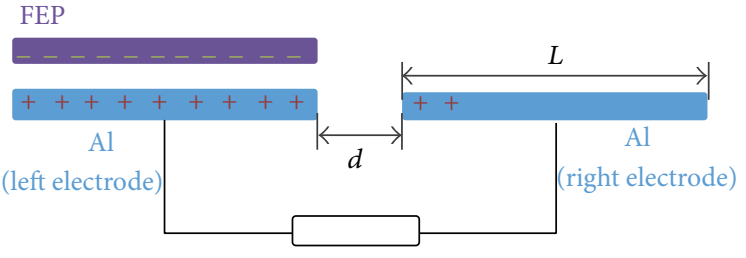

(c)

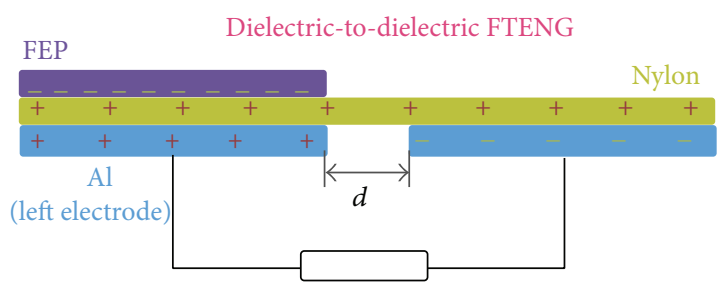

(d)

FIGURE 5: (a) Schematic illustration of a free-standing triboelectric-layer mode TENG [47]. (b) Working principle of a free-standing triboelectric-layer mode TENG. Sketches of (c) a conductor-to-dielectric TENG and (d) a dielectric-to-dielectric TENG.

Here, to characterize the electrical output, short-circuit current $\left(I_{\mathrm{sc}}\right)$, and open-circuit voltage $\left(V_{\mathrm{oc}}\right)$ of the TENG, a mechanical shaker is used to apply impulse impact. With a contacting force of $10 \mathrm{~N}, V_{\mathrm{oc}}$ and $I_{\mathrm{sc}}$ of the output terminals are displayed in Figures 6(d) and 6(e), respectively. It can be observed that $V_{\text {oc }}$ is switched between zero and a plateau value, respectively (Figure 6(d)), and $I_{\mathrm{sc}}$ exhibits AC behavior, and it can get up to $160-175 \mu \mathrm{A}$ (Figure 6(e)). As the force reaches $500 \mathrm{~N}$, the output gets the saturated value, producing peak $I_{\text {sc }}$ of $1.2 \mathrm{~mA}$ (Figure 6(f)). This is because the full contact area of the two materials can be achieved by a larger force. Resistors are used as external loads to further investigate the output power of the TENG. When the contacting force is $500 \mathrm{~N}$, a power output of $0.42 \mathrm{~W}$ can be achieved (Figure 6(g)). To demonstrate that the fabricated TENG can be used as a power source to light up commercial LEDs, 600 green LEDs are connected to the TENG. As illustrated in Figure 6(h), 600 green LEDs can be simultaneously lit up. These results suggest the possibility of device for a sustainable power source. 


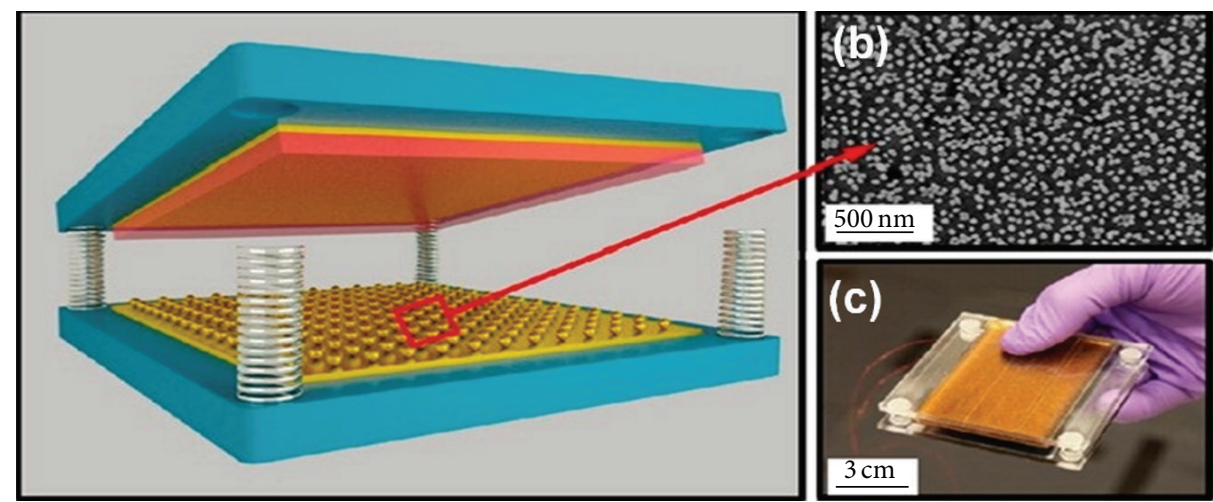

(a)

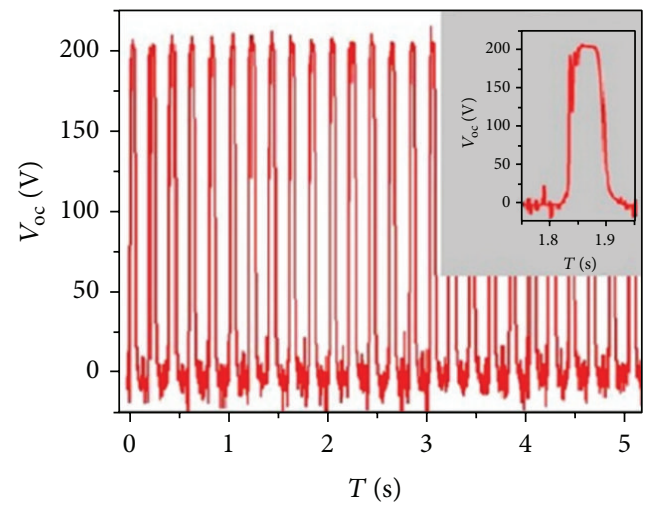

(d)

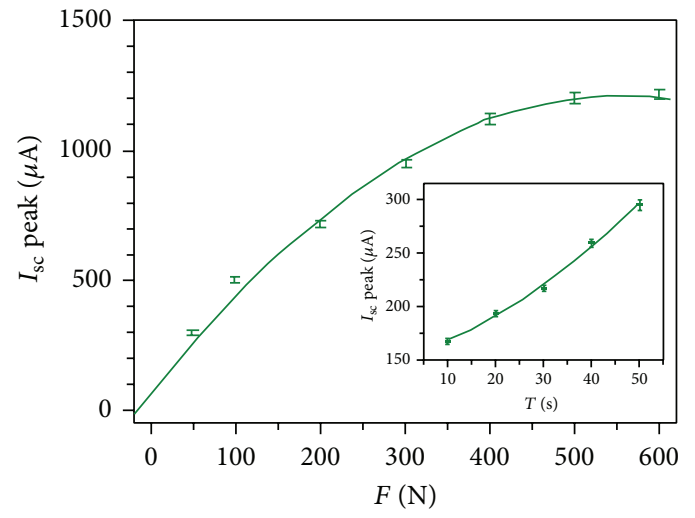

(f)

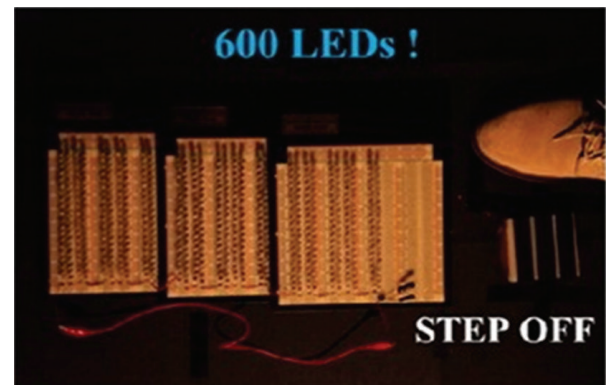

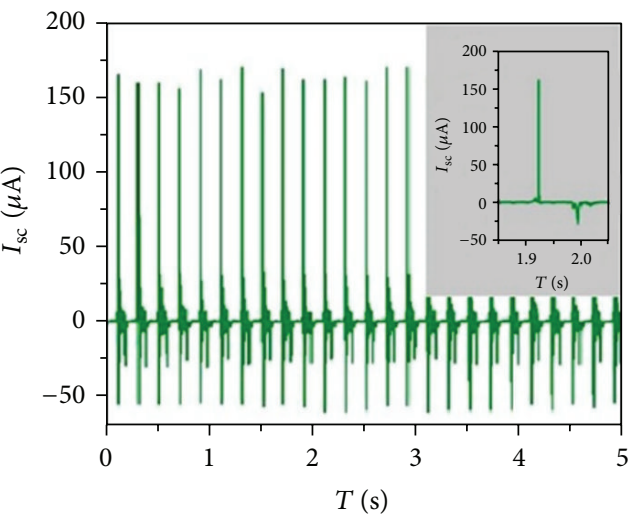

(e)

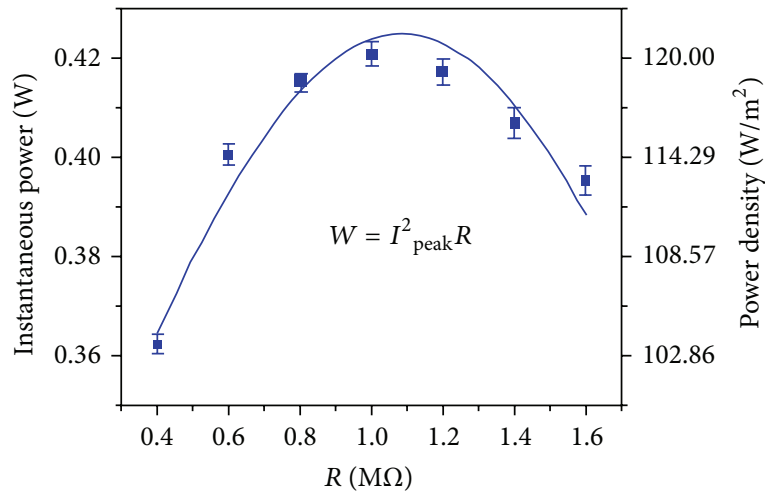

(g)

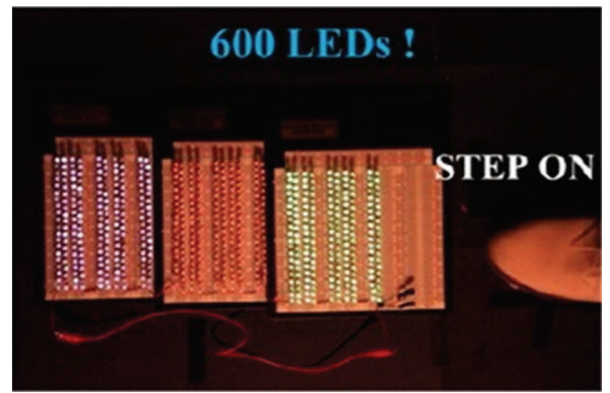

(h)

Figure 6: (a) Structural design of the TENG [14]. (b) A top-view SEM image of gold nanoparticles covered on gold surface. (c) A photograph showing the fabricated device. (d) The output open-circuit voltage $\left(V_{\mathrm{oc}}\right)$ and $(\mathrm{e})$ the output short-circuit current $\left(I_{\mathrm{sc}}\right)$ at contacting force of $10 \mathrm{~N}$. (f) Dependence of the short-circuit current $\left(I_{\mathrm{sc}}\right)$ on the contacting force. (g) Dependence of the peak power output on the resistance of the external load. (h) When footstep falls off/on the TENG. 


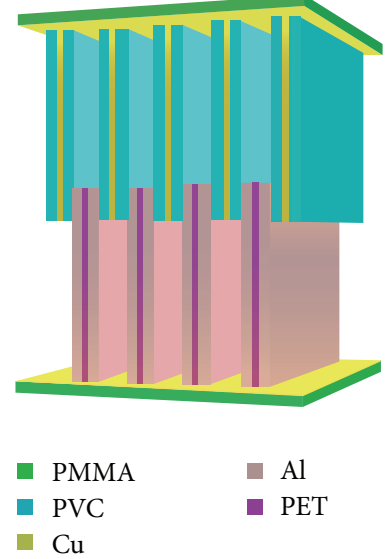

(a)

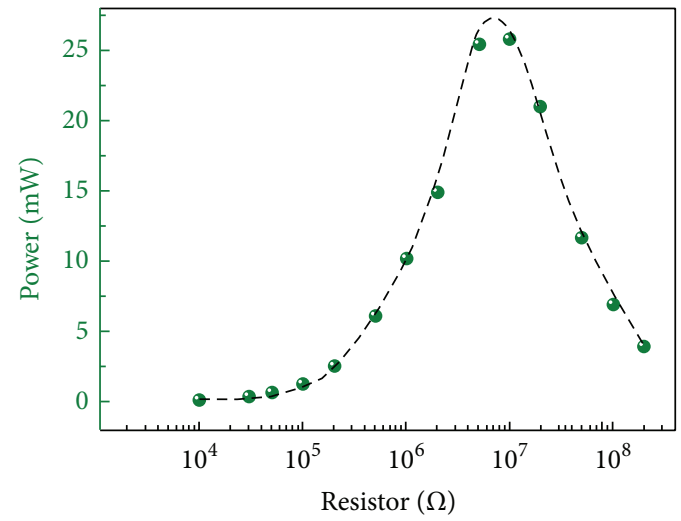

(c)

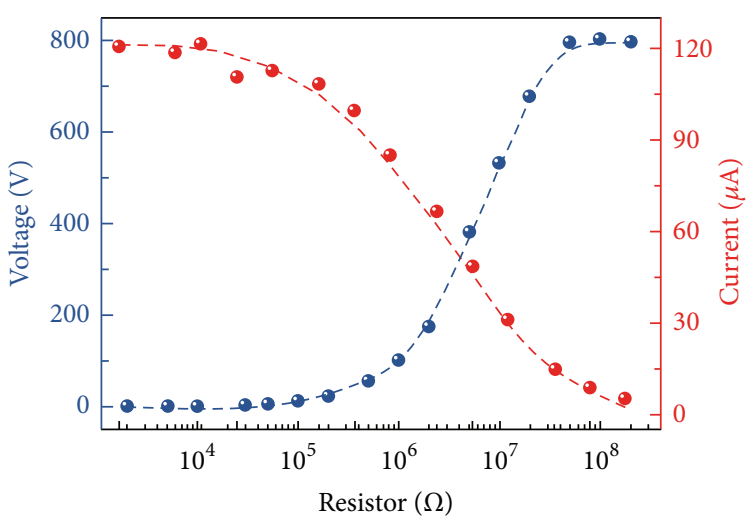

(b)

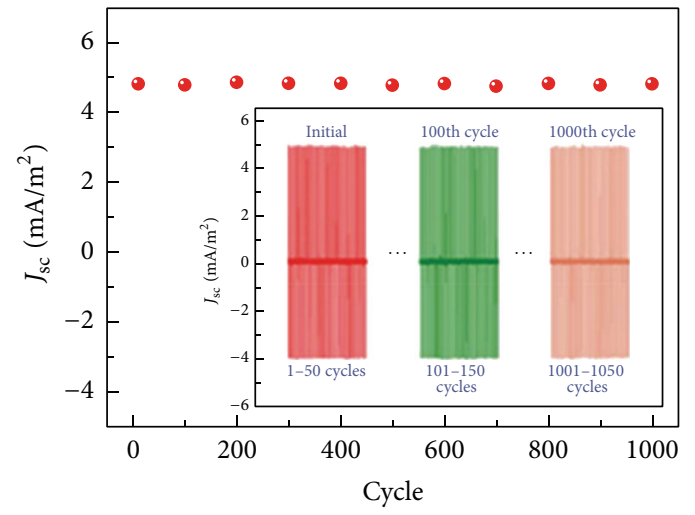

(d)

Figure 7: (a) Schematic of the device structure [55]. (b) The output voltage (blue) and current (red) and (c) the power on the resistance of the external load. (d) The output stability performance of the TENG.

Furthermore, a three-dimensional triboelectric nanogenerator (3D TENG) based on an in-plane sliding mode is also demonstrated for large scale energy harvesting [55]. The 3D TENG with layer-by-layer stacked polyvinyl chloride (PVC) and aluminum as friction materials is schematically illustrated in Figure 7(a). The efficient fiction area is largely increased owing to the multilayered structure. The opencircuit voltage of approximately $800 \mathrm{~V}$ is observed with an output current of $120 \mu \mathrm{A}$ from the 3D TENG based inplane sliding mode, as demonstrated in Figure 7(b), and the current reduces with the increasing load resistance while the output voltage shows a reversal trend. The output power of the 3D TENG is also plotted as a function of external resistance as shown in Figure 7(c). The results show that the output power is maximized at around $27 \mathrm{~mW}$ at an external resistance of about $8 \mathrm{M} \Omega$. In this case, the 3D TENG can drive the microelectronics or other sensors. Notably, there are no significant differences in the short-circuit current density measured from the TENG over 1000 cycles (Figure 7(d)); thus the stability of the 3D TENG is great for applications and this novel device presents a practically effective approach in producing electricity at a large scale.
3.2. Enhancing Output Current for Energy Harvesting. There is a general challenge for the application of the TENG, which is the fact that the output current is usually low. Synchronizing the outputs of all multiple units can potentially be utilized to enhance the instantaneous output current of energy harvesting devices $[53,56]$.

In this regard, an innovative design of TENG integrated rhombic gridding was developed as a cost-effective and robust approach to address this issue because of the multiple unit cells connected in parallel [56]. The integrated rhombic gridding structure of TENG is schematically shown in Figure $8(\mathrm{a})$, in which the total number of unit cells is determined by the following equation:

$$
N_{\text {total }}=2 n^{2}
$$

where $n$ is the number of unit cells along the edge length. The PET with a thickness of $600 \mu \mathrm{m}$ is employed as the substrate. In each unit cell, an aluminum thin film with nanopores serves not only as a triboelectric surface but also as an electrode. PTFE coated copper is employed as another contact surface. 


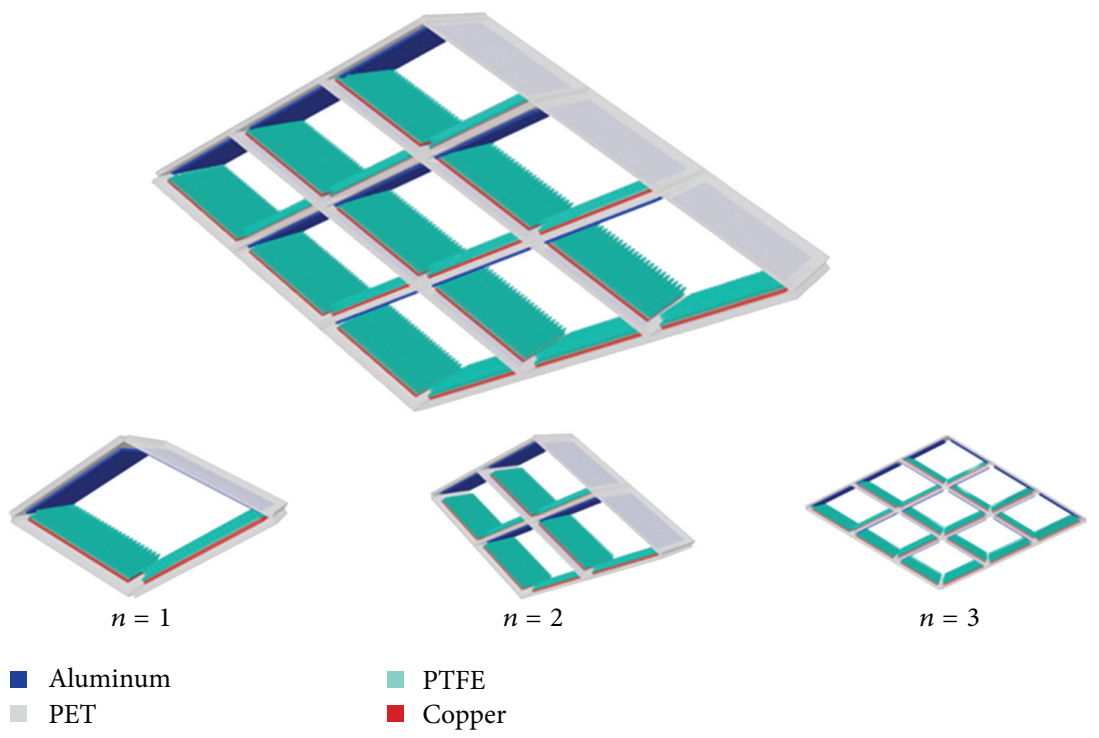

(a)

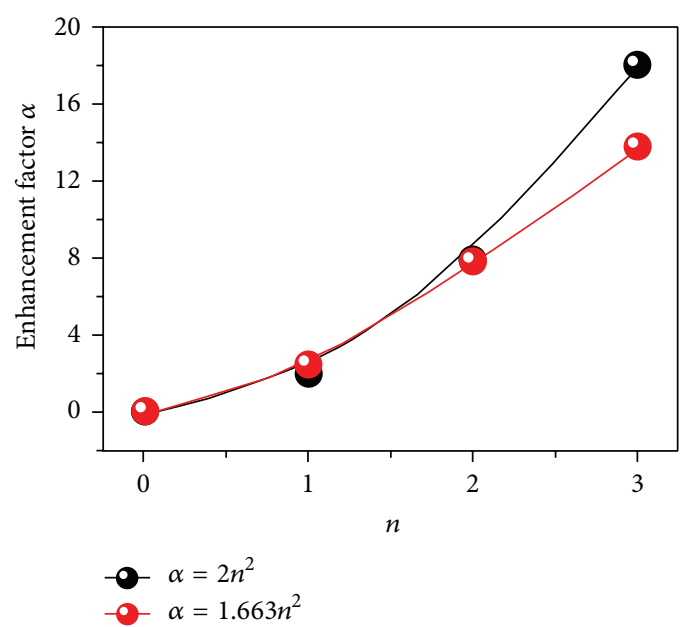

(b)

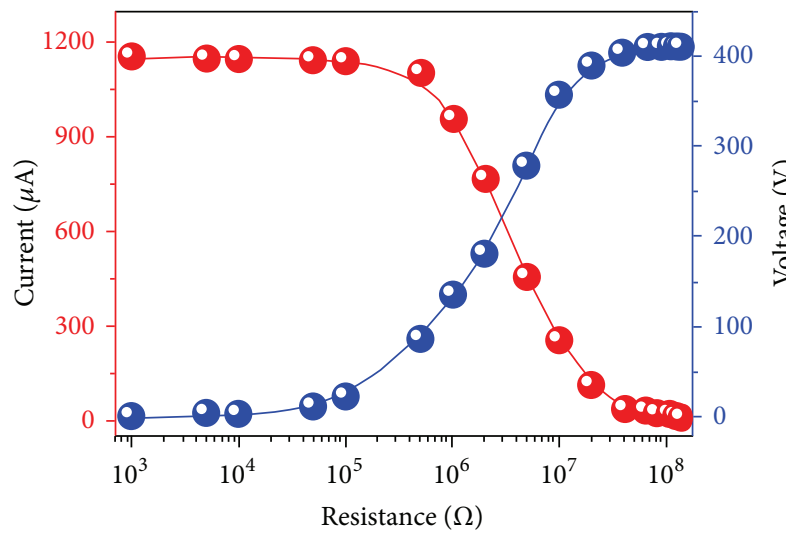

(d)

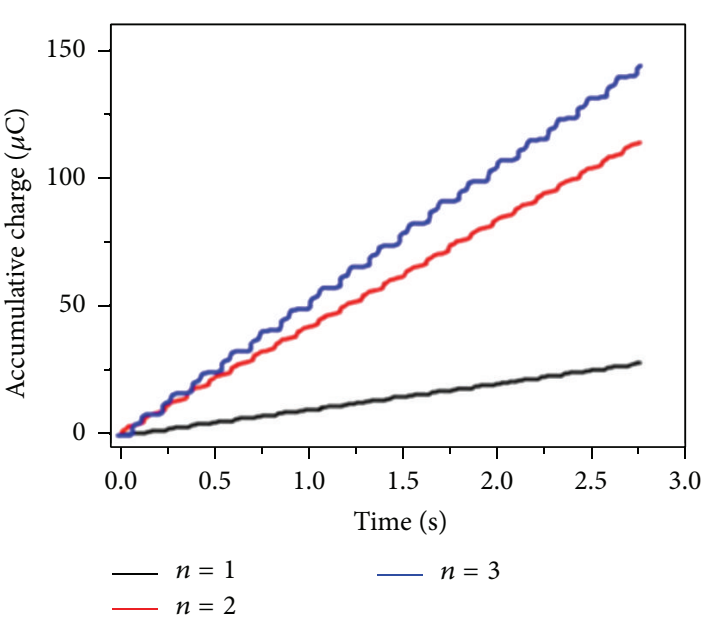

(c)

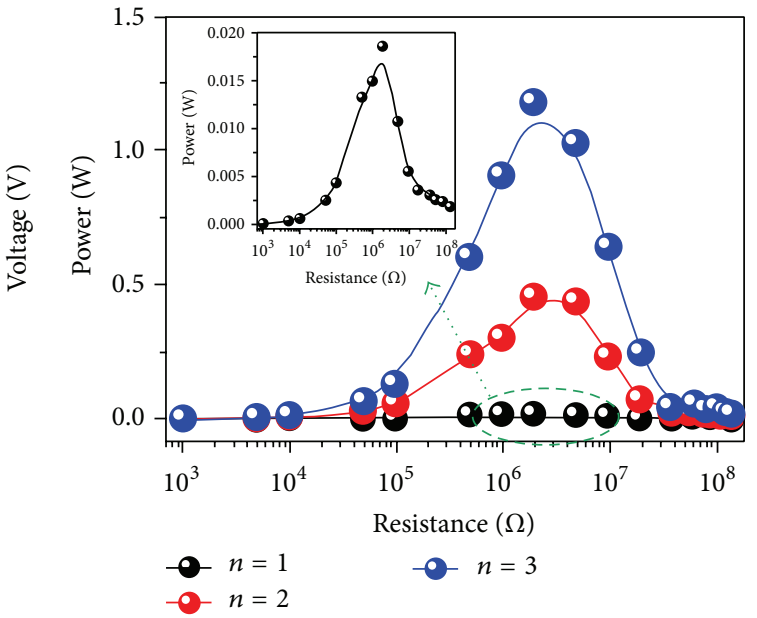

(e)

FIGURE 8: (a) Schematic diagram of the TENG and when $n=1,2,3$, respectively [56]. (b) The current's enhancement factor $\alpha$ increases with $n$. (c) Accumulative inductive charges for $n=1,2,3$, respectively. (d) Dependence of the voltage and current output on the external load resistance. (e) Dependence of the peak power output on the resistance of the external load with $n=1,2,3$. 


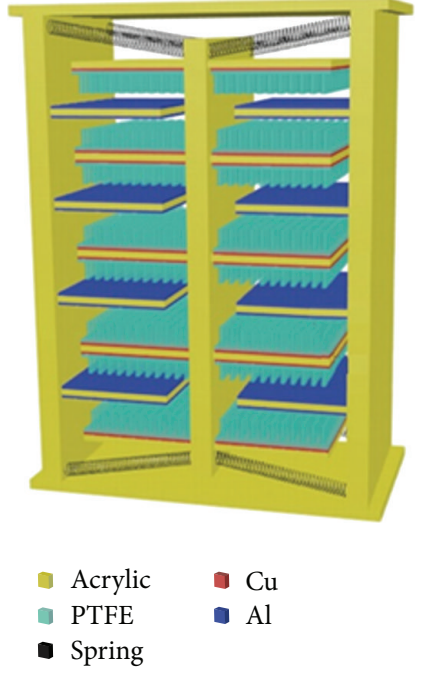

(a)

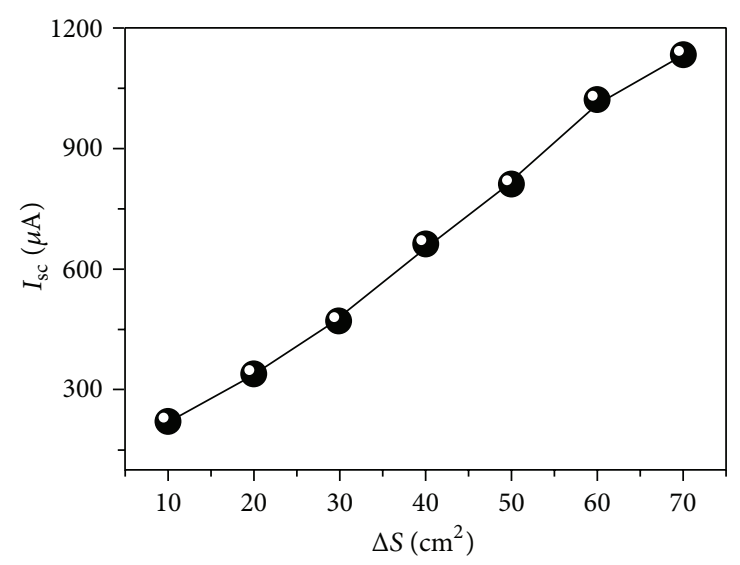

(c)

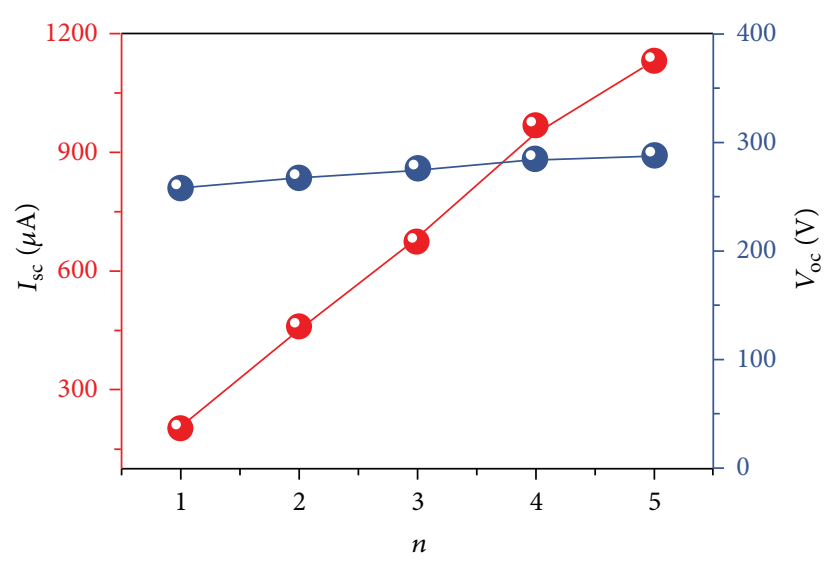

(b)

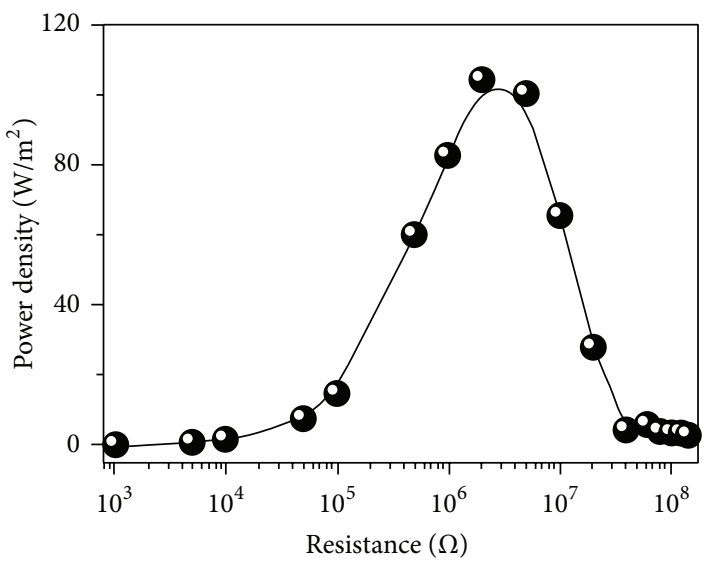

(d)

FIGURE 9: (a) Schematic diagram of the multilayered stacked TENG [53]. (b) Dependence of the voltage and current output on the number of pinned fingers. (c) Dependence of the short-circuit current versus the effective contact area $(\Delta S)$. (d) Dependence of the peak power density on the external load resistance.

As shown in Figure 8(b), $\alpha$ is the current enhancement factor which is a function of the number of unit cells, given by $\alpha=b n^{2}$. Due to the nonideal experimental factors, such as humidity, fitting coefficient $b$ is a value of 1.66. The observed results of enhancement factor are considerably approaching the ideal value of $2 n^{2}$, and thus the innovative structure is able to greatly enhance the current output. As displayed in Figure 8(c), the accumulative induced charges dramatically increase with increasing $n$ and when $n=3$, further revealing that the novel integrated rhombic gridding structure can dramatically improve the electric output. In addition, the effective electrical power of TENG is closely related to the external load, as shown in Figure 8(d), and the currents decrease with the increase of load resistance while the output voltages show a reversal trend. Moreover, the peak power dramatically increases with $n$, and the peak power of $1.17 \mathrm{~W}$ can be obtained at $n=3$ (Figure 8(e)).

Moreover, to enhance the instantaneous output current of energy harvesters, a 3D stack integrated TENG is presented
[53], which has a multilayered structure with acrylic as supporting substrates, as schematically illustrated in Figure 9(a). The number of the units in a 3D TENG is the critical factor that affects the output current, which can be expressed as the following equation:

$$
N=4 n,
$$

where $n$ is the total number of pinned fingers of a 3D TENG. To further investigate the effect $n$ on the electric output, the voltage output and current are measured, as depicted in Figure 9(b). The voltage output remains almost constant for different number of pinned fingers $n$, while the current output increases linearly with the number of pinned fingers, reaching about $1.2 \mathrm{~mA}$. This experimental result contributes to the operating synchronicity of all units. Likewise, the current output increases with the increase of the effective contact area $\Delta S$, as displayed in Figure 9(c). And the peak power density of the 3D TENG can reach up to $104.6 \mathrm{Wm}^{-2}$ with $n=5$ 


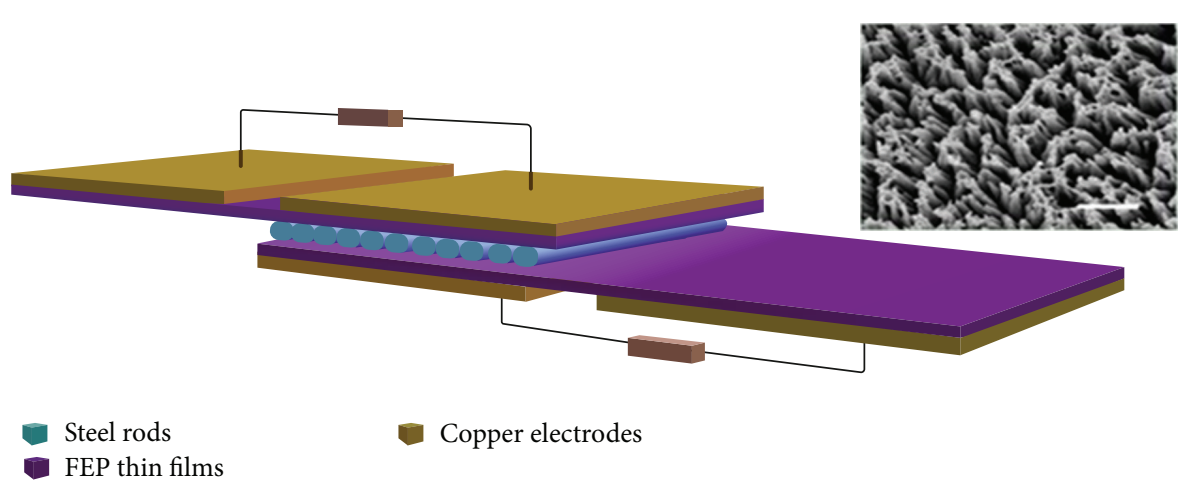

(a)

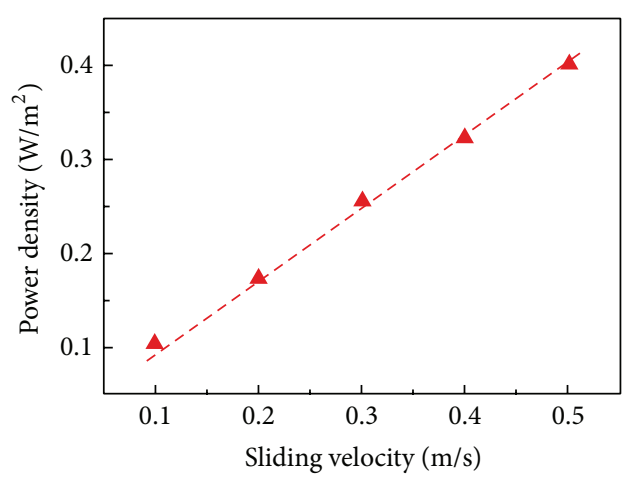

(b)

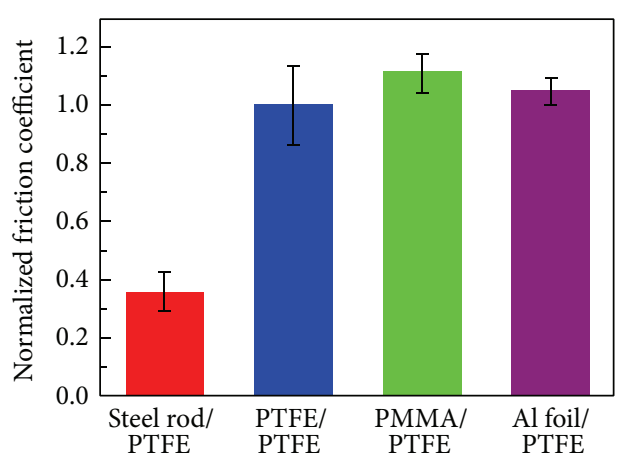

(d)

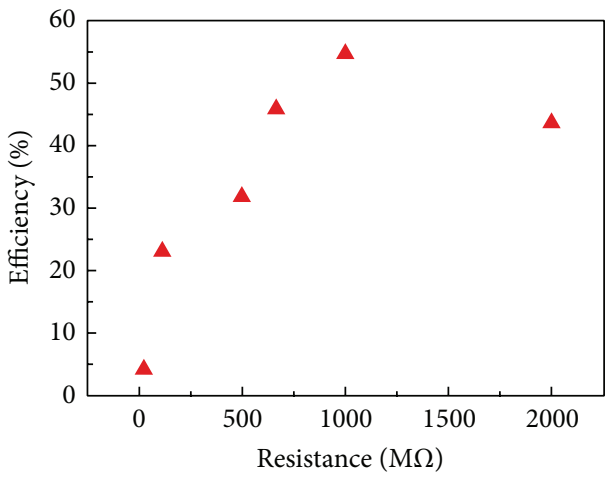

(c)

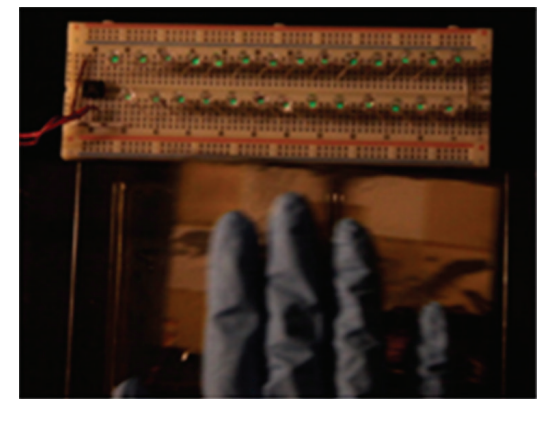

(e)

Figure 10: (a) Schematic diagram of the device structure [60]. (b) Dependence of the output power density of the TENG on the sliding velocity. (c) Conversion efficiency versus the resistance of the external load. (d) Normalized friction coefficient between different kinds of materials. (e) Photograph of commercial LEDs lit up simultaneously by the TENG.

(Figure 9(d)). As a consequence, the output current is greatly enhanced due to the integrated multilayered structure.

\subsection{Enhancing Energy Conversion Efficiency For Energy Har-} vesting. Many endeavors have been devoted to development of delivering high energy conversion efficiency of the TENG [57-62]. An advanced structural design of the TENG which generates periodically changing triboelectric potential and alternating currents between electrodes was demonstrated [60].

The TENG has a multilayered structure. It consists of a group of rolling steel rods sandwiched by two layers of FEP thin films and copper which is coated onto the FEP film as back electrodes, as illustrated in Figure 10(a). As the top layerFEP moves from the left end to the right end of the bottom layer-FEP, the steel rods would also move from the left part to the right part of the bottom layer-FEP, which will introduce triboelectric charges on both surfaces of the FEP thin films and lead to the change of potential difference between each pair of electrodes on the back of the FEP layer, driving the electrons to flow in the resistance of the external load. Figure 10(b) shows the maximum output power increases with increasing velocities from 0.1 to $0.5 \mathrm{~m} / \mathrm{s}$. And the energy conversion efficiency can be calculated to be $\sim 55 \%$ when the resistance of the external load is matched with the internal 

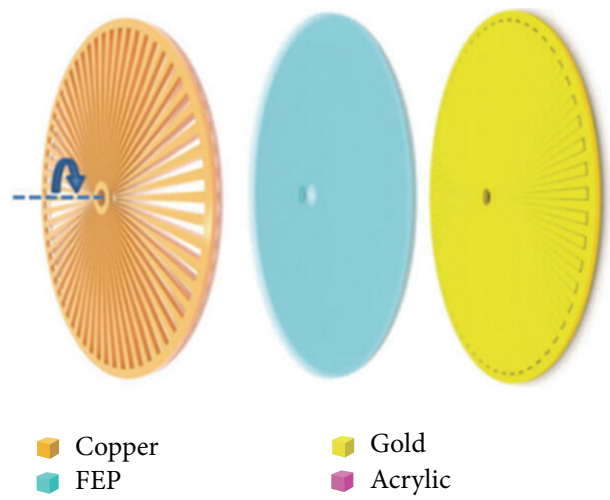

(a)

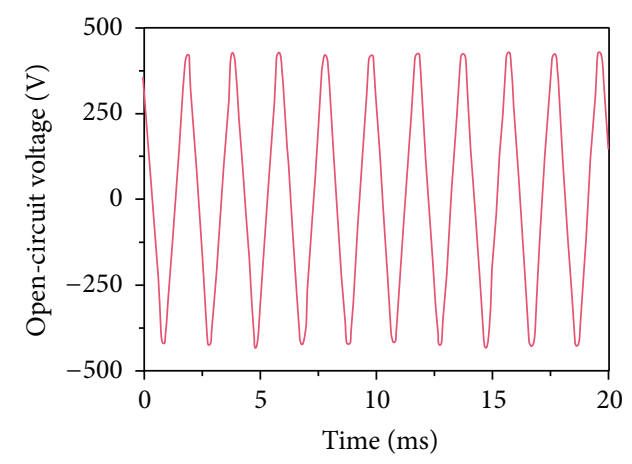

(c)
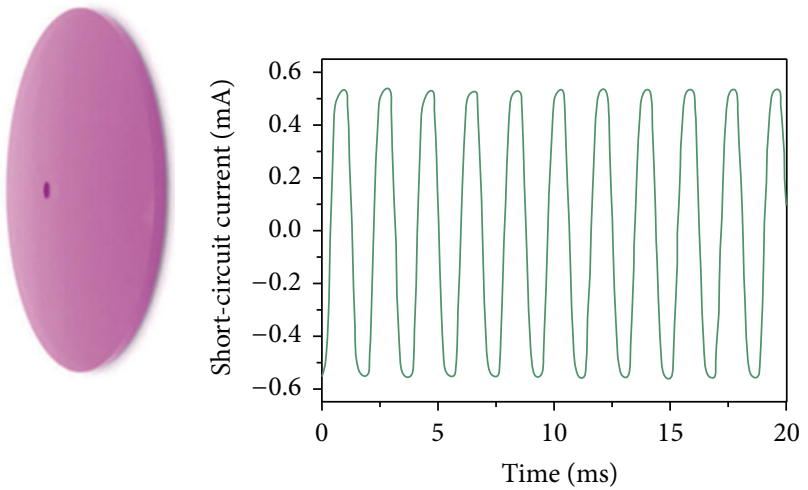

(b)

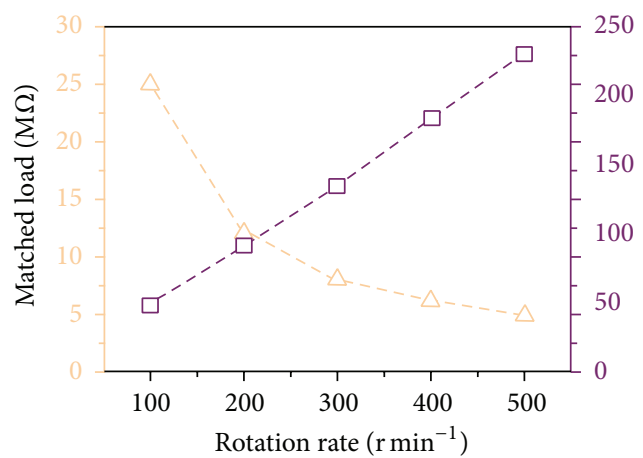

(d)

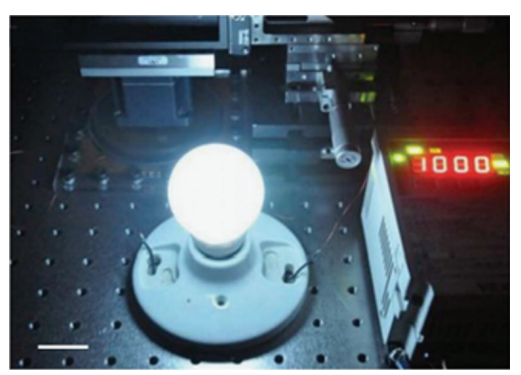

(e)

FIGURE 11: (a) Device structure of the TENG [24]. (b) Short-circuit current and (c) open-circuit voltage at a rotation rate of $500 \mathrm{r}$ min $^{-1}$. (d) Matched load and average power versus the rotation rate. (e) A photo of G16 globe lit up.

impedance of the TENG (Figure 10(c)). The high energy conversion efficiency of the TENG can be owing to the low frictional coefficient of the rolling motion. As for the result of normalized friction coefficient, the frictional coefficient of the rolling rod structure is lower than that of the planar structures, as shown in Figure 10(d). The TENG can also power portable electronics, like light-emitting diodes (Figure 10(e)).

In order to further increase energy conversion efficiency, many advanced structures have been developed with multilayered materials. Zhu et al. demonstrated a planarstructured TENG with two radial-arrayed fine electrodes [24], which is composed of mainly two parts, that is, a rotator and a stator, as shown in Figure 11(a). The working principle of the TENG is based on the free-standing triboelectric-layer mode, generating alternating currents between electrodes.
When the TENG works at a rotating rate of $500 \mathrm{r} \mathrm{min}^{-1}$, the continuous short-circuit current can reach $0.5 \mathrm{~mA}$ with a constant frequency of $500 \mathrm{~Hz}$ (Figure 11(b)). Figure 11(c) shows the measured open-circuit voltage of the TENG, where the peak-to-peak value can reach up to about $870 \mathrm{~V}$ at the same frequency. To validate the performance of the TENG, Figure 11(d) depicts the matched load and output power curves at higher rotation rates, and it is found that the matched load significantly reduces with the increase of the rotation rate, while the output power is the proportional relationship with the rotation rate. Furthermore, the average output power of $1.5 \mathrm{~W}$ can be obtained at a rotation rate of $3000 \mathrm{r} \mathrm{min}^{-1}$ and the efficiency can reach as high as $\sim 24 \%$. The TENG can also be applied to power some electronics such as lighting up a white globe light, as illustrated in Figure 11(e). 

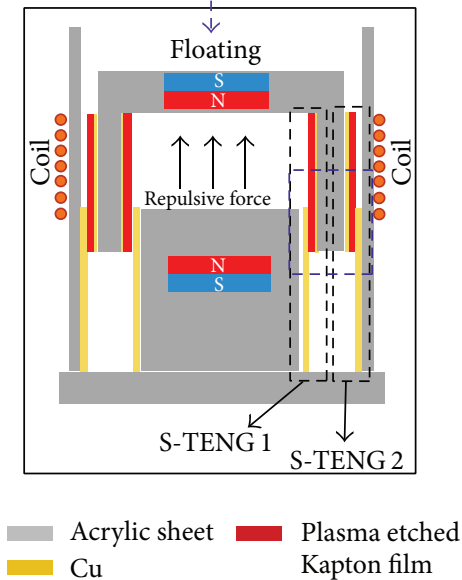

(a)

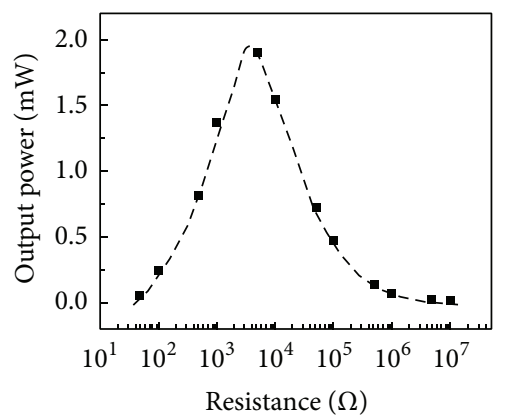

(d)

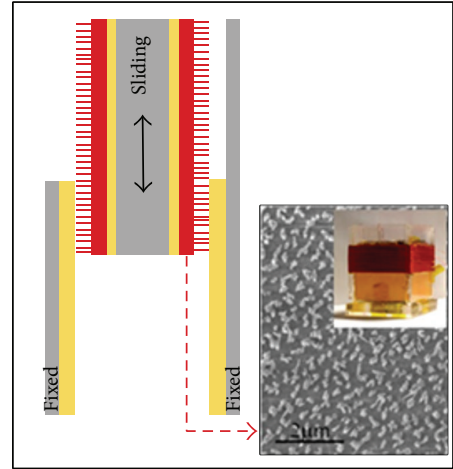

Plasma etched Kapton film

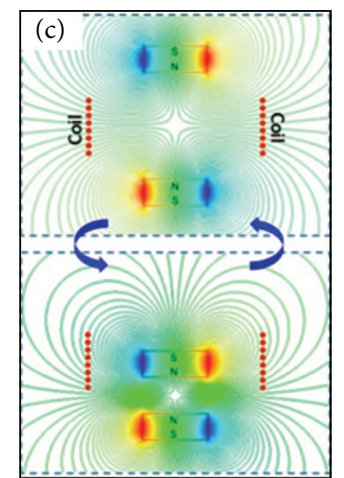

(b)

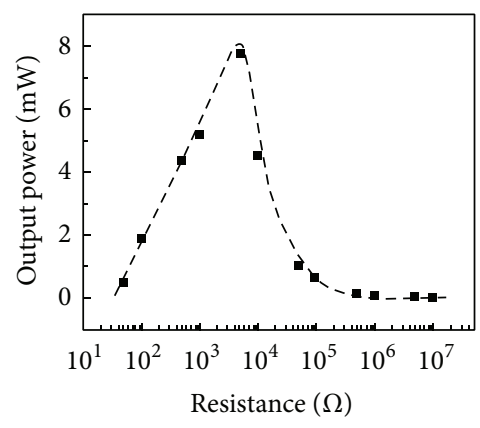

(e)

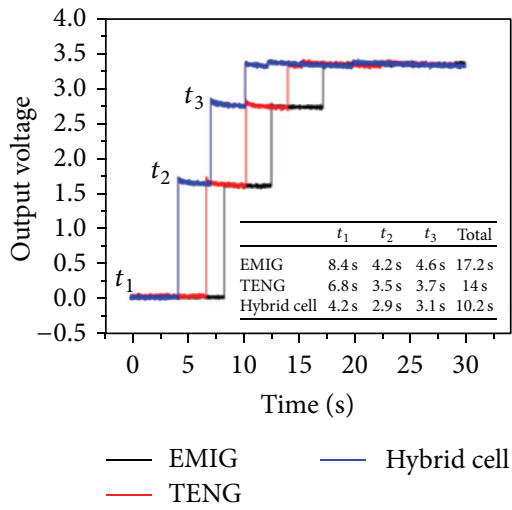

(f)

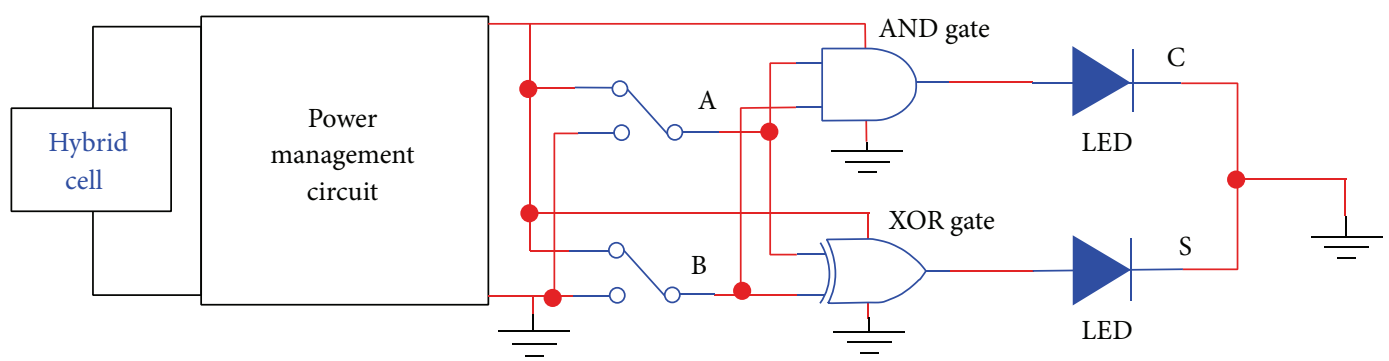

(g)

FIgURE 12: (a) Schematic diagram of the cross section of the hybrid cell [64]. (b) Sketch of the sliding mode TENG structure. The inset is an image of as-fabricated device. (c) Simulations of distribution of magnetic field in vibration. ((d) and (e)) Dependence of the peak power output on the resistance of the EMIG and TENG. (f) The capacitors charged by the EMIG, TENG, and hybrid cell. (g) Schematic diagram showing the hybrid cell to drive a half-adder circuit.

\section{Hybridization of Triboelectric Nanogenerators with Other Types of Energy Harvesters}

The energy available such as light, mechanical, thermal, and even chemical energy can also be scavenged as a sustainable power source. Thus, there are many other types of power generators, such as solar cells [63-65], electromagnetic generators [66], and electrochemical cells [67]. By integrating TENGs with one of the above-mentioned generators, more electricity can be largely generated, increasing the total output power of the energy device to meet the power needs of some electronic devices [68-71].

\subsection{Hybridized Electromagnetic-Triboelectric Nanogenerator.} In order to enhance the overall power output, many attempts have been developed. A hybridized mechanical energy harvesting technology may be utilized to enhance the total output performance. Hu et al. developed a hybrid cell composed of a TENG and an electromagnetic generator (EMG) for scavenging energy [66]. Figure 12(a) illustrates a schematic 


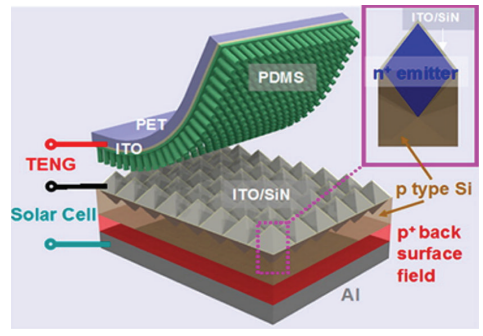

(a)

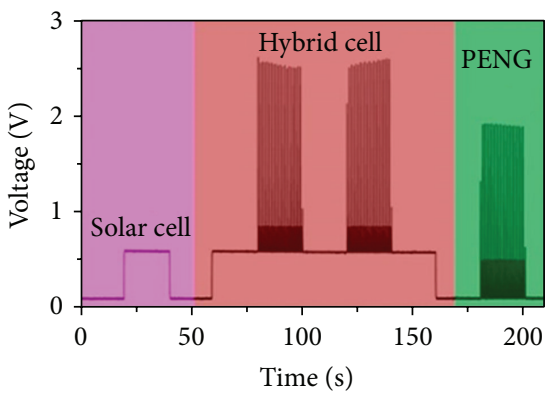

(e)

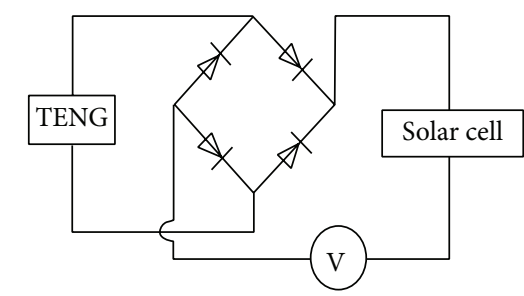

(b)

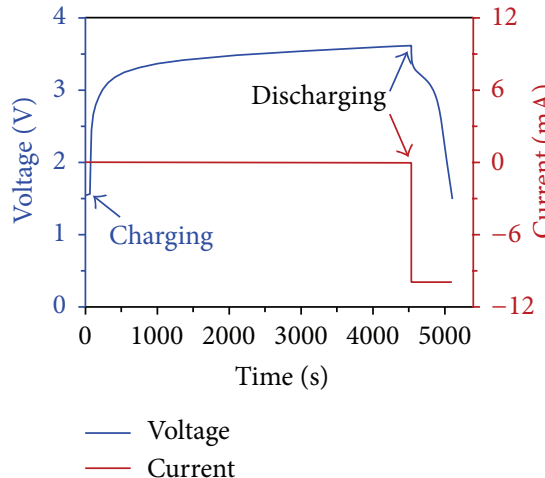

(f)

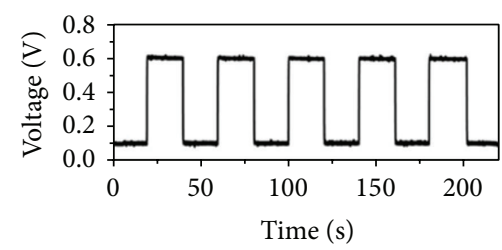

(c)

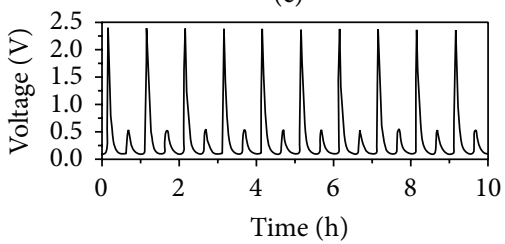

(d)

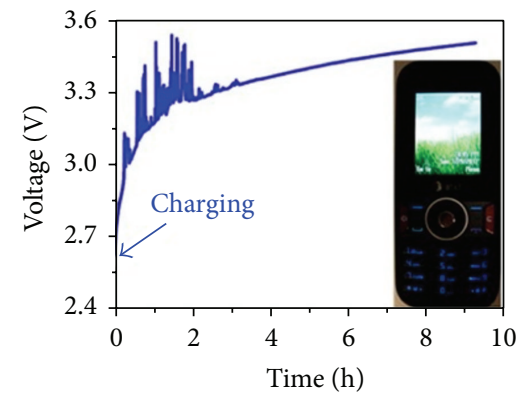

(g)

Figure 13: (a) Schematic showing the structure of hybrid energy cell [68]. (b) Schematic illustration of the bridge rectification circuit. (c) Output voltage of the Si solar cell. (d) Rectified output voltage of the TENG. (e) Output voltage of the hybrid solar cell and TENG. (f) The hybrid energy cell charging and discharging curves of a Li-ion battery. (g) A Li-ion battery of a cell phone charged by the hybrid energy cell.

diagram of a hybridized electromagnetic-triboelectric nanogenerator, which consists of a copper wire and a smaller hollow cube with an opening bottom floating in a larger hollow cube and a solid cube fixed on the bottom of the larger hollow cube. The Kapton films are coated on the inner and outer side wall surfaces of the smaller hollow cube, and the copper foil is attached on the side wall surfaces of the solid cube and the lower part of the inner surfaces of the larger hollow cube. Figure 12(b) illustrates the enlarged center working part. And there are two magnets integrated in the smaller hollow cube and the solid cube, respectively.

When there is a mechanical disturbance, the TENGs operate in the vertical contact-separation mode and in-plane sliding mode. Likewise, the magnetic flux in the coil will be changed, and an electric output will be generated in the coil because of electromagnetic induction. Figure 12(c) shows the induced magnetic field distribution of a copper wire versus the position of the top magnet by simulation. The TENG has a very high output voltage but low output current with large output impedance; however, the EMG can produce high current but low voltage with small output impedance. Thus, transformers are applied to the measurement system. Figures $12(\mathrm{~d})$ and 12(e) illustrate the rectified output power signals of the TENG and EMG with transformer; the output curves of the EMGs are similar to TENGs, but the peak output power of EMGs is lower than TENGs. To demonstrate that the hybridized nanogenerator has a better output performance than individual energy harvesting units, different generators are used to get a $3.3 \mathrm{~V}$ regulated output voltage. As shown in Figure 12(f), the shortest charging time is obtained by the hybridized nanogenerator, indicating that the hybridized nanogenerator has a much better charging performance than that of the individual energy harvesting unit where the charging voltage can reach up to $3.3 \mathrm{~V}$ in about $10.2 \mathrm{~s}$. Furthermore, the hybrid cell after regulation can also drive a half-adder circuit, as demonstrated in Figure 12(g). Hence, the hybridized electromagnetic-triboelectric nanogenerator has potential applications for energy harvesting and promotes the progress of energy harvesting.

4.2. Hybridized Nanogenerator by Integrating TENG with Solar Cell. To scavenge different kinds of energy, a hybrid energy cell which is fabricated directly for simultaneously scavenging both mechanical and solar energies was demonstrated, while the hybridized nanogenerator can play the role of a sustainable power source for the batteries.

Figure 13(a) is the schematic diagram of the device, which is based on the hybridization of the TENG with Si solar cell [72]. The Si solar cell is composed of Al electrode, p+ layer, $\mathrm{p}$ type $\mathrm{Si}$ layer, $\mathrm{n}+$ emitter layer, $\mathrm{SiN}$ film, $\mathrm{Ag}$ grids, and the transparent ITO electrode. The protection layer for $\mathrm{Si}$ microstructure is replaced by a flexible transparent 
film of nanowires coated with an ITO electrode. Thus, the PDMS thin film coated ITO electrode and the conducting Si surface compose the TENG. The PDMS layer plays dual role of the effective triboelectric-layer for the TENG and the high transparent protection layer for the Si solar cell. Figure 13(c) displays that the output voltage of Si solar cell shoots up to $0.6 \mathrm{~V}$, where the corresponding output current reaches $18 \mathrm{~mA}$. There is a bridge rectification circuit used to convert the AC into DC signals (Figure 13(b)), and the rectified output voltage of $2.5 \mathrm{~V}$ for TENG can be obtained, as shown in Figure 13(d). Figure 13(e) shows the output voltage for each generator both individually and in serial connection when the rectified TENG and the solar cell are connected in series (Figure 13(b)). The peak output voltage of the hybrid nanogenerator can be enhanced owing to the TENG, and it can be further improved to $12 \mathrm{~V}$. Figure 13(f) depicts a typical charging and discharging cycle of Li-ion battery. The voltage of the battery increased from 1.54 to $3.60 \mathrm{~V}$ in about $1.3 \mathrm{~h}$. Under a constant current of $10 \mathrm{~mA}$, the discharging of the battery can last for about $580 \mathrm{~s}$ before it is discharged back to its original voltage of $1.54 \mathrm{~V}$. That is, the hybridized nanogenerator can be used to charge up Li-ion battery. Figure 13(g) shows that Li-ion battery of a commercial cell phone is charged from 2.63 to $3.50 \mathrm{~V}$ in about $9 \mathrm{~h}$, further indicating the battery that can be used for some personal electronics.

\section{Applications}

As a new power generation technology, the TENG makes it possible to utilize the energy scavenged from the vast environment to directly drive the electronic devices. The formed self-powered systems can be applied to various applications such as personal medical networks, self-powered active sensors, health monitoring, acoustic sensing, homeland security, and even remote monitoring [15, 73-91].

5.1. 3D Energy Harvesting. The triboelectric nanogenerator has become an attractive concept for energy harvesting; however, most of the TENGs can only work at a single direction of ambient vibrations, which limits their applications in realworld environments. To overcome this issue, Yang et al. have presented a 3D triboelectric nanogenerator based on the coupling of the triboelectrification and the electrostatic induction, which can harvest random energy in multiple directions [15].

A schematic diagram of the $3 \mathrm{D}$ TENG is shown in Figure 14(a); the designed TENG consists of a cylindroid core, three springs, triboelectric materials, electrodes, and the acrylic substrate. In the top of the core, a copper electrode is sandwiched by an iron mass and a layer of PTFE film, and the iron mass is attacked by the three springs. An aluminum thin film with nanopore modification is adhered onto the acrylic substrate (Figure 14(b)), which not only serves as an electrode but also serves as the other contact surface. Figure 14(c) shows the photograph of the TENG. The 3D TENG shows that it can work in multiple directions relying on a hybridized mode of both vertical contact-separation and in-plane sliding. To study the output performance of the $3 \mathrm{D}$
TENG, Figure 14(d) demonstrates the measured open-circuit voltage of the 3D TENG, where the voltage reaches $123 \mathrm{~V}$ under out-of-plane excitation. Besides, a plateau value of $142 \mathrm{~V}$ can be obtained under in-plane excitation, as depicted in Figure 14(e). Furthermore, there is a bandwidth up to $75 \mathrm{~Hz}$ for 3D TENG operating under out-of-plane excitation, as illustrated in Figure 14(f). It can be found that the 3D TENG has a significant output performance and an extremely wide working bandwidth in different vibration direction. To further investigate the practical application in our surrounding, the 3D TENG is used to scavenge the line vibrational energy. As demonstrated in Figures 14(g) and 14(h), respectively, it can be seen that 40 commercial LED bulbs are lit up simultaneously (Figure $14(\mathrm{~g})$ ). To systematically study the electric output, Figure 14(h) shows the voltage values under different line swing amplitudes. We can see that the output voltage increases dramatically with the increase of the swing amplitudes. In addition, to get a visualization of the 3D TENG powering electronics, the 3D TENG can be also mounted on a bicycle wheel to scavenge the rotation energy, and 30 commercial LEDs are connected in series and lit up simultaneously, as shown in Figure 14(i). Likewise, the 3D TENG can be further applied to powering personal medical networks, infrastructure monitoring, and more.

5.2. Energy Harvesting from Human Motion. As we all know, portable electronic devices are usually powered by traditional power supplies, such as batteries, which significantly limits the usage of personal electronics. Meanwhile using the TENG which converts the biomechanical energy from human motions into electricity is a possible approach to drive portable electronic devices [73-78]. Thus Bai et al. demonstrated a flexible multilayered triboelectric nanogenerator for harvesting biomechanical energy from human motions [79].

The basic structure of the integrated multilayered TENG is schematically illustrated in Figure 15(a). The Kapton film is employed as the framework of the TENG units, which is shaped into a zigzag structure through making deformations. In each unit, an aluminum foil and PTFE thin film coated aluminum are adhered onto both sides of the Kapton film as the two contact surfaces, respectively. The integrated multilayered TENG has significantly larger output power and Figure 15(b) shows the short-circuit current and the opencircuit voltage under different large contact force. It can be seen that both the short-circuit current and the open-circuit voltage increase with the enhancement of the large contact force owing to the increased contact area. Meanwhile, to characterize the robustness of the multilayered TENG, the TENG is measuring the electric output for every ten thousand impacts. As depicted in Figure 15(c), there is only slight decrease for the short-circuit current and the open-circuit voltage about $\sim 7 \%$ and $\sim 9 \%$, respectively. To study the ability of the TENG to drive electronic devices, a self-lighting shoe is presented. The TENG is assembled onto the shoe pad, as shown in Figure 15(d). Once triggered by normal walking, a total of 9 commercial LED bulbs are lit up simultaneously (Figure 15(e)), which demonstrates the ability of the multilayered TENG to harvest biomechanical energy from human motions to power personal electrics. 

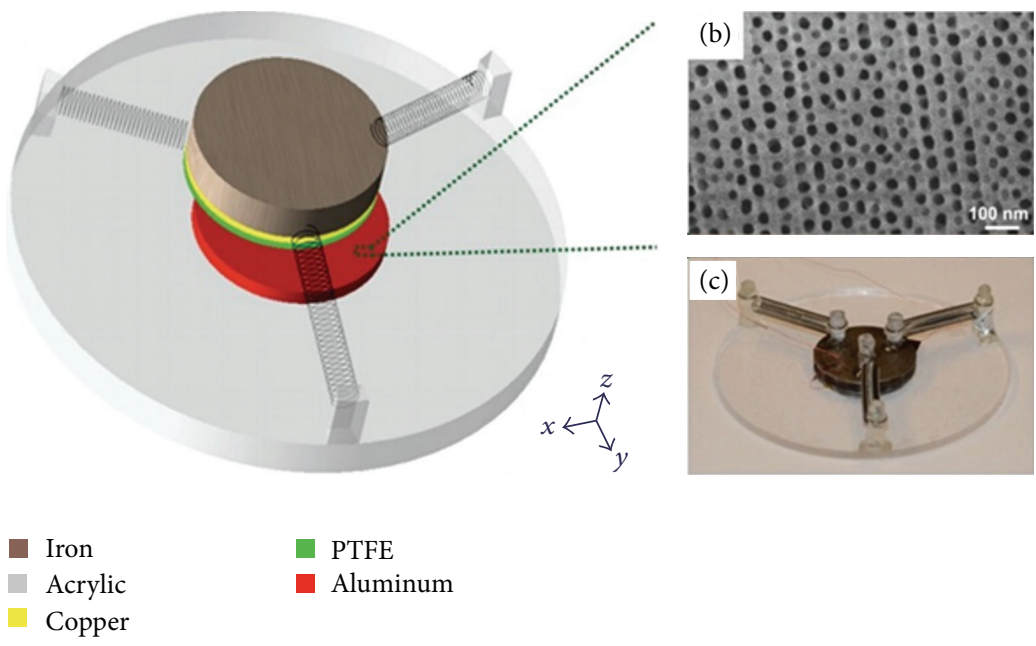

(a)

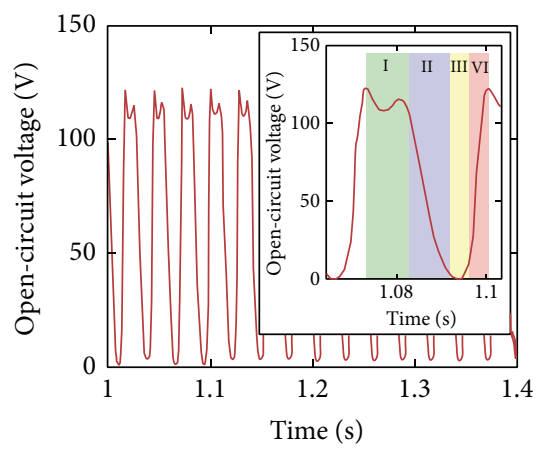

(d)

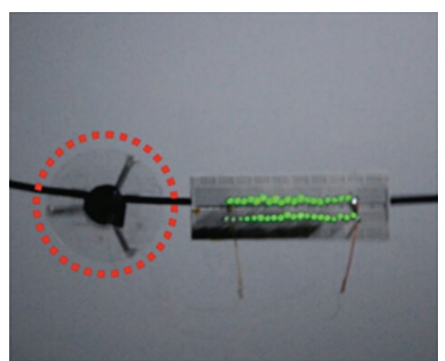

(g)

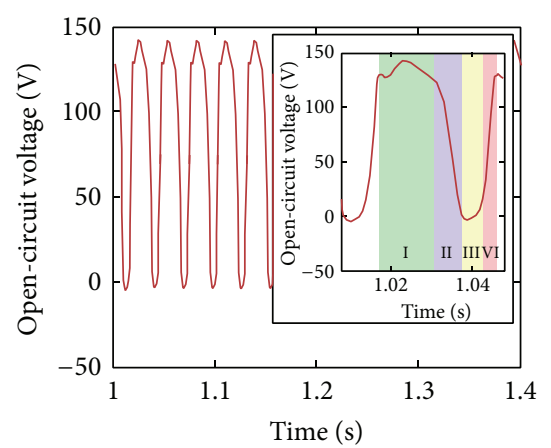

(e)

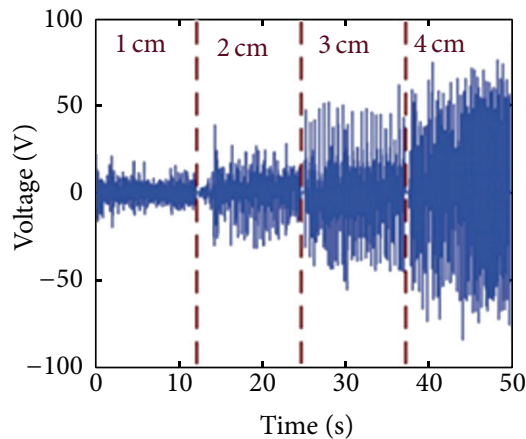

(h)

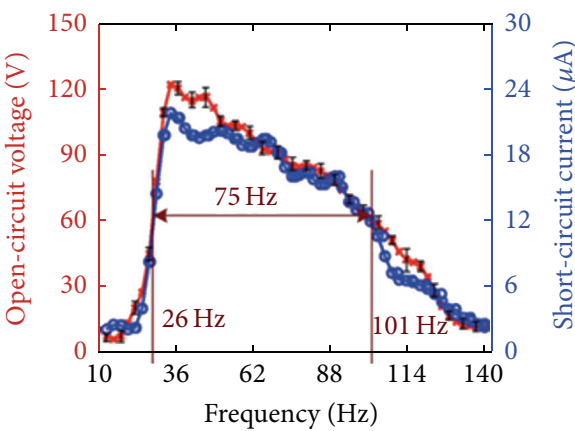

(f)

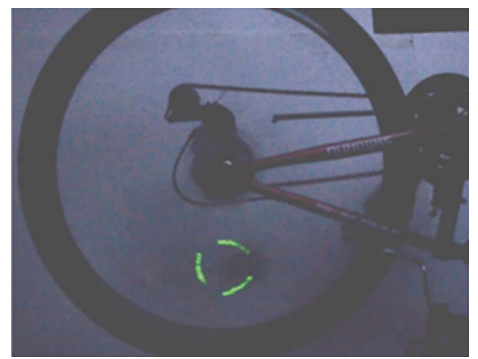

(i)

FIGURE 14: (a) Schematic diagram of a 3D triboelectric nanogenerator [15]. (b) SEM image of the aluminum film fabricated with nanostructures nanopores. (c) A photograph of an as-fabricated 3D TENG. Dependence of the open-circuit voltage (d) under out-of-plane excitation and (e) at in-plane excitation angle of $180^{\circ}$. (f) Output voltages versus frequency under out-of-plane condition. (g) Photograph of energy harvesting from the line vibration. (h) Output voltage under different line swing amplitudes condition. (i) Photograph of energy harvesting from the rotation of a bicycle wheel.

As demonstrated in Section 3.2, the TENG can be also used to harvest energy from human motions [56], which comprises a supporting shelf, a TENG, four springs, and two long screw shanks, as illustrated in Figure 15(f). When a person walks, the TENG will produce electric output and the photograph of a fabricated device is demonstrated in Figure 15(g). Moreover, the energy harvested from human walking can light up 30 serial-connected commercial LEDs simultaneously, as shown in Figure 15(h). Therefore, the TENG is proved to be suitable for biomechanical energy harvesting. In addition, Yang et al. also developed a 3D TENG which can harvest energy from human motions [15].

5.3. Acoustic Energy Harvesting. The acoustic energy that always exists in our daily life and environments has been overlooked as a power source such as speech, music, or even 


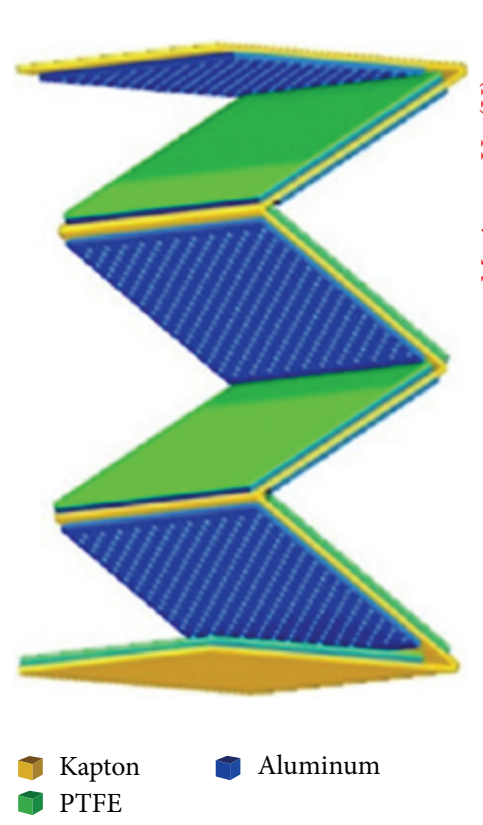

(a)

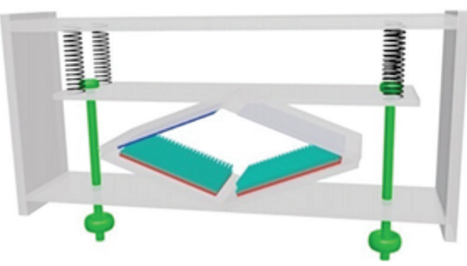

(f)

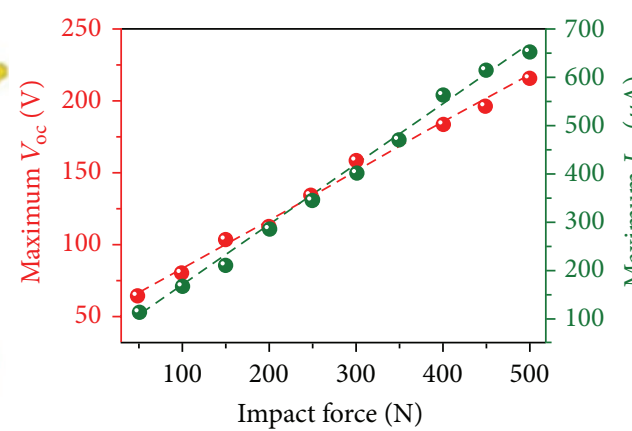

(b)

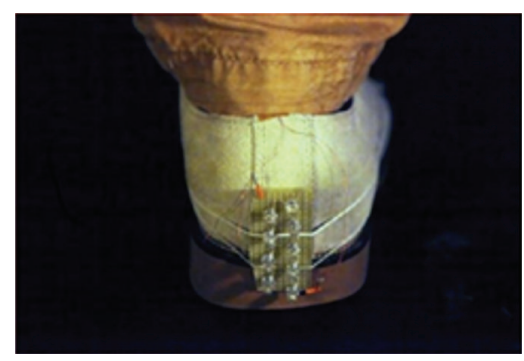

(d)

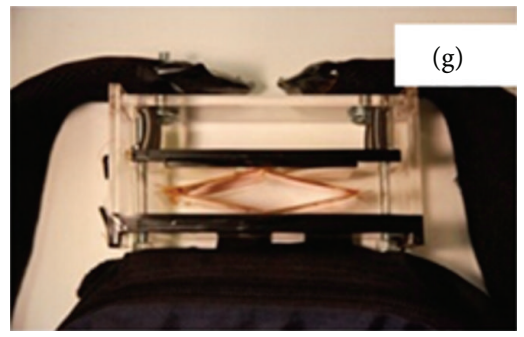

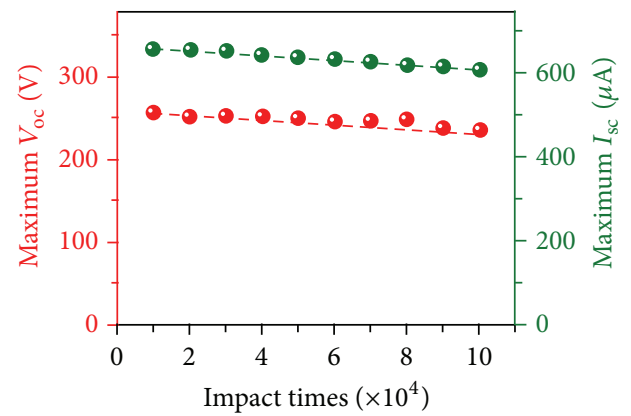

(c)

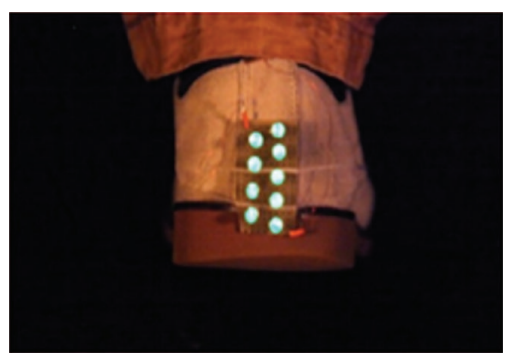

(e)

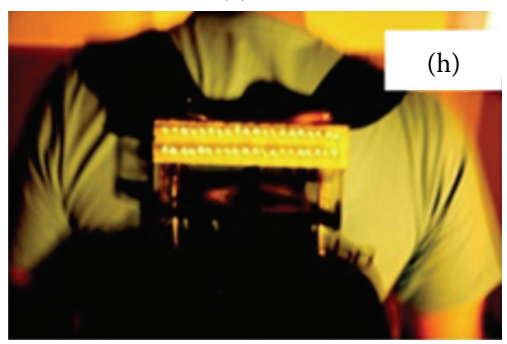

FIGURE 15: (a) Schematic diagram of an integrated multilayered TENG [71]. (b) Dependence of the short-circuit current and the open-circuit voltage versus the contact force. (c) Durability of the integrated multilayered TENG. (d) Photograph of an as-fabricated self-lighting shoe. (e) Photograph of the self-lighting shoe under normal walking. (f) Schematic diagram of a self-powered backpack [56]. (g) Photograph of an as-fabricated self-powered backpack. (h) Photograph of the backpack under normal walking.

noise. How to turn acoustic energy from speech, music, or noise into electrical power has been an attractive research. Thus, a triboelectrification-based thin film nanogenerator for harvesting acoustic energy from ambient environment is demonstrated to provide a practical method [80].

Figure 16(a) shows a schematic diagram of the nanogenerator, which is based on a Helmholtz cavity with a size-tunable narrow neck. The core of the nanogenerator (a cross-sectional view) is illustrated in Figure 16(b), in which aluminum film with nanopores serves as one contact surface and an electrode. To investigate the output performance, the output voltage and current of the TENG are measured upon connecting to an external load resistor. As the results displayed in Figure 16(c), the voltage amplitudes through the load will generally increase, while the current follows a reverse trend because of the Ohmic loss. In addition, the instantaneous power density generated from the TENG can reach a maximum value of $60.2 \mathrm{~mW} / \mathrm{m}^{2}$ at a resistance of $6 \mathrm{M} \Omega$, and it can simultaneously drive 17 commercial LED bulbs, as depicted in Figure 16(d). Moreover, the output voltage can also serve as sound signal transmitted. Parts (e1) and (e2) of Figure 16(e) are the reconstructed signal of sound and the corresponding short-time Fourier transform, respectively. Thus, the TENG can be developed as a self-powered microphone for sound recording, as shown in Figure 16(f). Therefore, the results suggest that acoustic energy can be one of the promising energy sources with highly efficient generators.

Besides, Fan et al. demonstrated a novel design of a rollable, paper-based TENG for harvesting acoustic energy [81]. Figure 16(g) shows the ultrathin TENG with a multilayered structure, consisting of a layer of multiholed paper coated with copper serving as an electrode layer and a thin PTFE membrane. The inset of Figure 16(h) is the photograph of the real device. The TENG has a large electric output and various applications; it can be seen that maximum output power density of $121 \mathrm{~mW} / \mathrm{m}^{2}$ with the corresponding volume power density of $968 \mathrm{~W} / \mathrm{m}^{3}$ is obtained at $800 \mathrm{~K} \Omega$, as demonstrated in Figure 16(h). Furthermore, it has the capability of harvesting acoustic energy from portable electronics. As shown in Figure 16(i), using the acoustic energy from a cell phone, the capacitor is charged from $0 \mathrm{~V}$ to $1.8 \mathrm{~V}$ within $17 \mathrm{~s}$. It is anticipated that the TENG harvesting acoustic energy can 


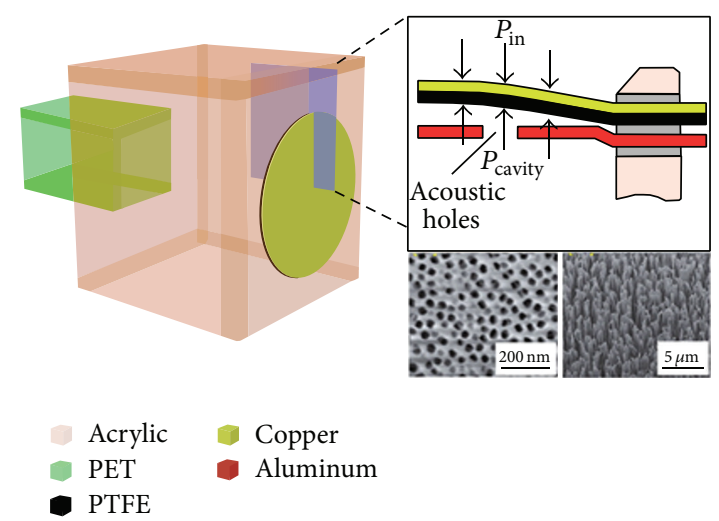

(a)

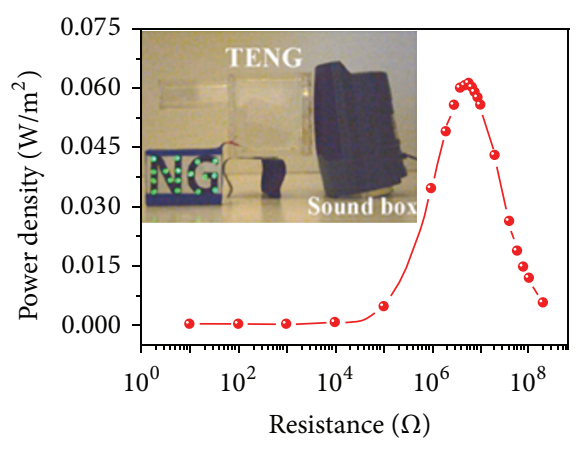

(d)

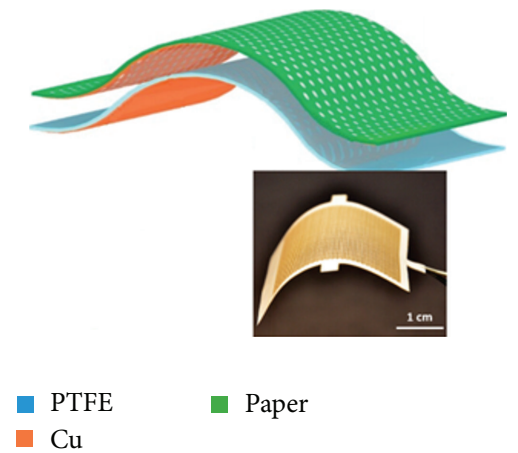

(g)

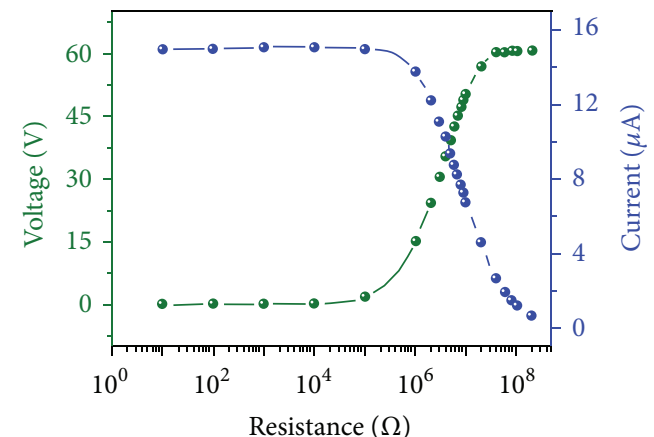

(c)

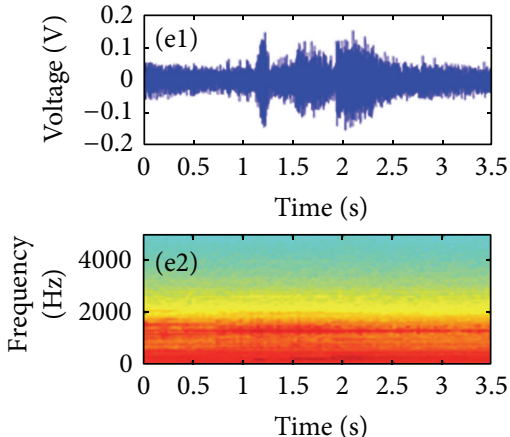

(e)

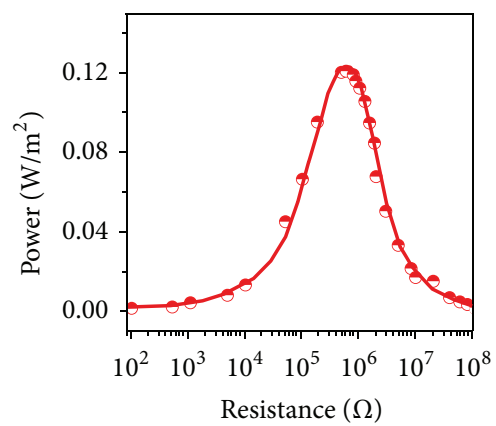

(h)

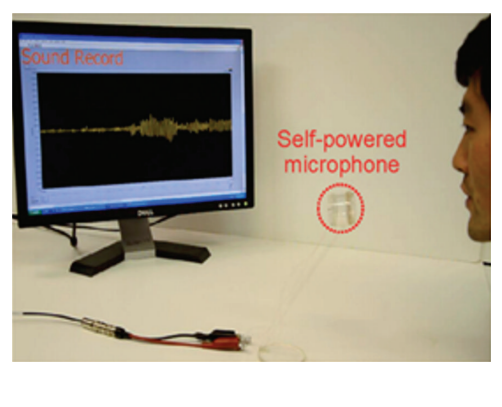

(f)

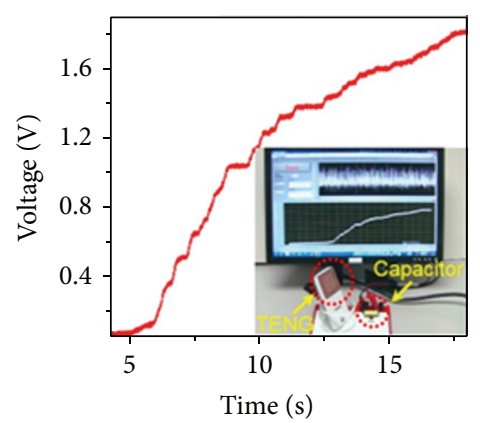

(i)

FIGURE 16: (a) Device structure of the organic film TENG [72]. (b) Cross-sectional view of the TENG. (c) Dependence of the short-circuit current and the open-circuit voltage and (d) the peak power output on the external load resistance. (e) The signal of sound waveform and short-time Fourier transform. (f) Photograph of a self-powered microphone for sound recording. (g) Schematic diagram of an as-fabricated ultrathin paper-based TENG [73]. (h) Dependence of the peak power output on the external load resistance. (i) A $2 \mu$ F capacitor charged by the THEG from the cell phone.

be applied to the applications of theatric stage live recording, jet engine noise reduction, military surveillance, and more.

5.4. Water Wave Energy Harvesting. Water wave energy is very abundant and common for us, which has the features of high volume, large scale, being widely distributed, and more. Thus, many efforts have been devoted to converting various types of water energy into electricity $[85,86]$ and there are also several challenges for commercialization up to now. Hu et al. reported a TENG with $3 \mathrm{D}$ spiral structure, which has the capability of efficiently scavenging water wave energy in our surrounding.

Figure 17(a) illustrates the structure of the TENG with a vertical contact-separation mode [87]. The basic structure of the TENG is composed of a 3D spiral structure with seismic mass at the bottom and a Kapton film serves as lower contact surface. An aluminum film coated on the seismic mass serves as the upper surface. The inset of Figure 17(a) is a photograph of the real device. When the spiral oscillates in response to the water agitation, electricity will be generated. The output 


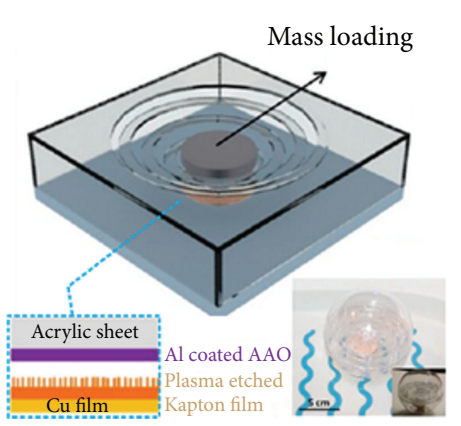

(a)

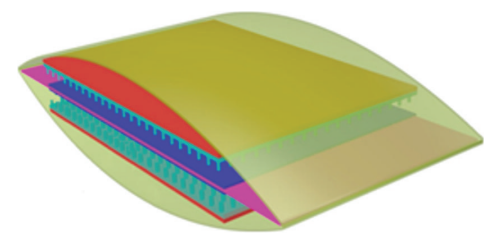

- $\mathrm{Cu}$

- $\mathrm{Al}$

- PET

(d)

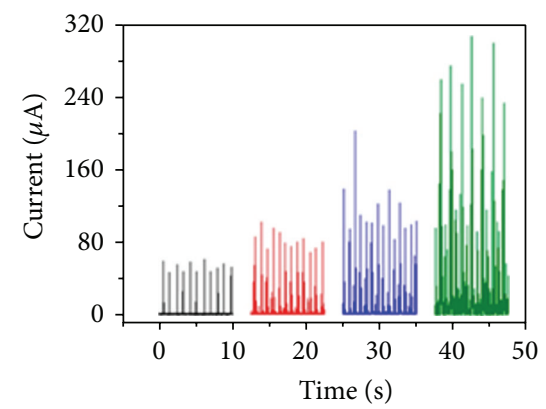

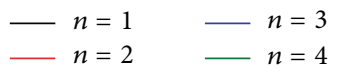

(g)

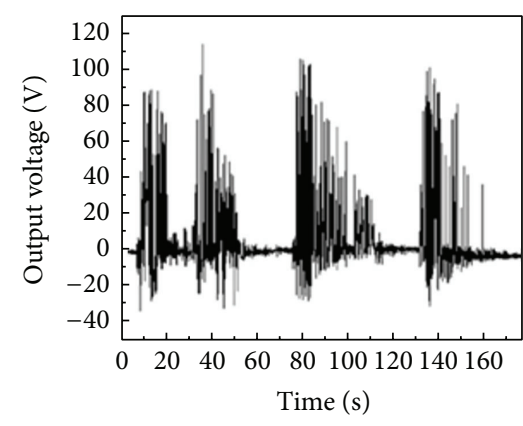

(b)

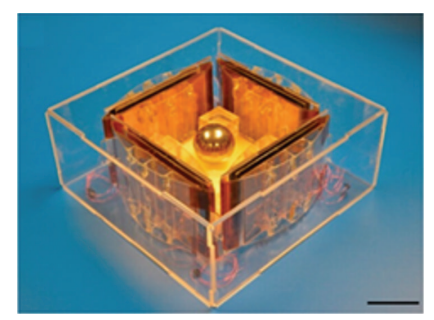

(e)

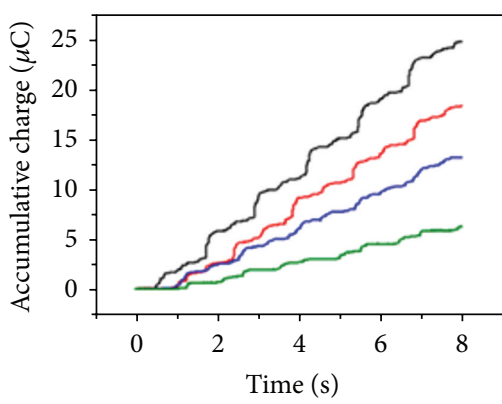

$\begin{aligned} n=4 & -n=2 \\ n & =3 \quad-n=1\end{aligned}$

(h)

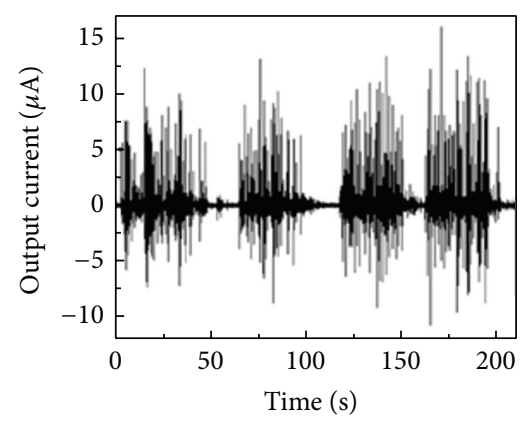

(c)

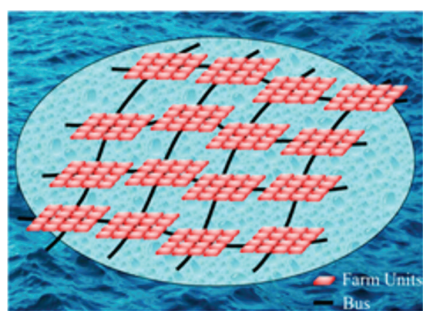

(f)

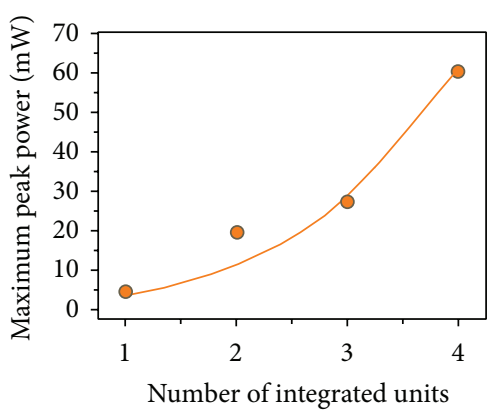

(i)

FIGURE 17: (a) Structural design of the TENG and a photo of the fabricated device [76]. (b) The open-circuit voltage and (c) the short-circuit current of the TENG. (d) Schematic of the fabricated minimum unit [77]. (e) Photo of a fabricated TENG. (f) Schematic for the configuration for harvesting water wave energy. (g) Rectified short-circuit current of the TENGs for different unit number. (h) Accumulative induced charges for different unit number. (i) Dependence of the peak power output on the resistance of the different unit numbers.

voltage and current of the TENG are depicted in Figures 17(b) and $17(\mathrm{c})$, respectively. From the results, it can be found that the device produces an output voltage peak of up to $110 \mathrm{~V}$ and the output current of up to $15 \mu \mathrm{A}$, which can light up some commercial LED bulbs as the buoy sways with the water waves. This approach might provide the possibility for the sustainable energy harvesting from water waves.

Another basic prototype of water-TENG is demonstrated for harvesting large scale water wave energy [91], as schematically illustrated in Figure 17(d). The basic unit of the TENG is composed of arch-shaped top and bottom plates and multilayer contact materials in it. Four basic units and a ball which plays the role of external vibration by gravity in the center of the device form a TENG, as shown in Figure 17(e). When the
TENG is driven by the water wave, the collision with the walls will drive the TENG generating electricity. The power output capability of the TENG is investigated with unit number $n=$ $1,2,3,4$, respectively. As demonstrated in Figure $17(\mathrm{~g})$, the output currents increase with the increase of unit numbers. Besides, similar trend also can be seen in Figure 17(h); the accumulative induced charges increase with elevated unit numbers. Moreover, a larger peak power output can be obtained owing to the increase of the unit numbers, which is plotted according to the integrated unit number in Figure 17(i). Thus the packaged TENG for collecting water wave energy is successfully demonstrated. Most importantly, large scale applications of TENGs as a sustainable power source can be adequately applied to harvest wave energy (Figure 17(f)). 


\section{Conclusions}

The discovery of the TENG takes a big step forward in the field of converting mechanical energy into electricity for meeting our energy demands. It is an exciting technology because of its high output intensity, high efficiency, light weight, low cost, easy fabrication, and small size. The TENG can effectively use mechanical energies in almost any form and any scale and provide a continuous direct current source to charging various commercial electronics under the four fundamental modes. Moreover, the TENG can be hybridized with other technologies such as the solar cell to simultaneously harvest multiple-type energies. And the hybridized nanogenerator is enabled to take advantages of both of their unique performances. Many practical applications have developed with great performance.

As to the future applications of TENGs, there are also several main issues on TENGs as a sustainable power source which we should pay more attention to. First, the fundamental mechanism of contact electrification remains to be investigated extensively. This phenomenon has been researched for a long time, which refers to the charge generation on the surface of the materials when they are brought into contact with different materials; several theories about the contact electrification have been proposed; however, there is no substantial conclusion achieved. Thus, the deepened understanding of contact electrification is vitally important to achieve a higher output of the TENG. Second, the output voltage of the TENG is very high, while its output current is low, which means that the TENG requires much higher optimum resistance than other harvesters. Voltage transformers may be an approach to reduce voltage and boost the current. In addition, the packaging of the TENG can be designed with many small size units to increase the output current and lower the output voltage without reducing the power. Third, the durability and output stability of the device should be improved. The performance of the surface of the materials may be changed for millions of cycles especially in contactsliding mode, which will affect the performance of the TENG. Through selecting new materials and designing advanced structure, the durability and output stability of the TENG can be enhanced. Fourth, a high efficiency power management circuit is required in order to provide power for electronics. The energy scavenging from the environment is unstable, unpredictable, and sometime intermittent, but the electronics need a regulated power source to work normally. So it is necessary to develop a high efficiency power management circuit to store the energy in a battery or capacitor and then provide power for the electronics forming a self-powered system.

The TENG has been a new paradigm in energy harvesting technologies for truly achieving sustainable and maintenance-free self-powered systems. It can be anticipated that through the worldwide efforts on TENGs as a sustainable power source they will soon be made commercialized products for the applications in Internet of things, mobile electronics, self-powered e-skins, fabric electronics, medical/ health monitoring, and environmental protection.

\section{Competing Interests}

The authors declare no competing financial interests.

\section{Acknowledgments}

This work was supported by the National Natural Science Foundation of China (nos. 61174017 and 51475060) and Chongqing Science and Technology Commission (cstc2013kjrc-qnrc40006).

\section{References}

[1] S. P. Beeby, M. J. Tudor, and N. M. White, "Energy harvesting vibration sources for microsystems applications," Measurement Science and Technology, vol. 17, no. 12, article no. R01, pp. R175R195, 2006.

[2] J. W. Matiko, N. J. Grabham, S. P. Beeby, and M. J. Tudor, "Review of the application of energy harvesting in buildings," Measurement Science and Technology, vol. 25, no. 1, Article ID 012002, 2014.

[3] E. Arroyo and A. Badel, "Electromagnetic vibration energy harvesting device optimization by synchronous energy extraction," Sensors and Actuators A: Physical, vol. 171, no. 2, pp. 266-273, 2011.

[4] J. Chen, D. Chen, T. Yuan, and X. Chen, "A multi-frequency sandwich type electromagnetic vibration energy harvester," Applied Physics Letters, vol. 100, no. 21, Article ID 213509, 2012.

[5] J. Yang, Y. Wen, P. Li, X. Bai, and M. Li, "Improved piezoelectric multifrequency energy harvesting by magnetic coupling," in Proceedings of the 10th IEEE SENSORS Conference 2011 (SENSORS '11), pp. 28-31, IEEE, Limerick, Irland, October 2011.

[6] J. Yang, Y. Wen, P. Li, X. Yue, and Q. Yu, "Energy harvesting from ambient vibrations with arbitrary in-plane motion directions using a magnetostrictive/piezoelectric laminate composite transducer," Journal of Electronic Materials, vol. 43, no. 7, pp. 2559-2565, 2014.

[7] Q. Yu, J. Yang, X. Yue et al., “3D, wideband vibro-impactingbased piezoelectric energy harvester," AIP Advances, vol. 5, no. 4, Article ID 047144, 2015.

[8] P. D. Mitcheson, P. Miao, B. H. Stark, E. M. Yeatman, A. S. Holmes, and T. C. Green, "MEMS electrostatic micropower generator for low frequency operation," Sensors and Actuators, A: Physical, vol. 115, no. 2-3, pp. 523-529, 2004.

[9] L. G. W. Tvedt, D. S. Nguyen, and E. Halvorsen, "Nonlinear behavior of an electrostatic energy harvester under wide-and narrowband excitation," Journal of Microelectromechanical Systems, vol. 19, no. 2, Article ID 5404427, pp. 305-316, 2010.

[10] J. Yang, Y. Wen, P. Li, X. Yue, Q. Yu, and X. Bai, "A twodimensional broadband vibration energy harvester using magnetoelectric transducer," Applied Physics Letters, vol. 103, no. 24, Article ID 243903, 2013.

[11] J. Yang, Q. Yu, J. Zhao et al., "Design and optimization of a bi-axial vibration-driven electromagnetic generator," Journal of Applied Physics, vol. 116, no. 11, Article ID 114506, 2014.

[12] K. Y. Lee, J. Chun, J.-H. Lee et al., "Hydrophobic sponge structure-based triboelectric nanogenerator," Advanced Materials, vol. 26, no. 29, pp. 5037-5042, 2014.

[13] G. Zhu, C. Pan, W. Guo et al., "Triboelectric-generator-driven pulse electrodeposition for micropatterning," Nano Letters, vol. 12, no. 9, pp. 4960-4965, 2012. 
[14] G. Zhu, Z.-H. Lin, Q. Jing et al., “Toward large-scale energy harvesting by a nanoparticle-enhanced triboelectric nanogenerator," Nano Letters, vol. 13, no. 2, pp. 847-853, 2013.

[15] J. Yang, J. Chen, Y. Yang et al., "Broadband vibrational energy harvesting based on a triboelectric nanogenerator," Advanced Energy Materials, vol. 4, no. 6, Article ID 1301322, 2014.

[16] S. Kim, M. K. Gupta, K. Y. Lee et al., "Transparent flexible graphene triboelectric nanogenerators," Advanced Materials, vol. 26, no. 23, pp. 3918-3925, 2014.

[17] W. Yang, J. Chen, G. Zhu et al., "Harvesting vibration energy by a triple-cantilever based triboelectric nanogenerator," Nano Research, vol. 6, no. 12, pp. 880-886, 2013.

[18] H. Zhang, Y. Yang, Y. Su et al., "Triboelectric nanogenerator for harvesting vibration energy in full space and as self-powered acceleration sensor," Advanced Functional Materials, vol. 24, no. 10, pp. 1401-1407, 2014.

[19] B. K. Yun, J. W. Kim, H. S. Kim et al., "Base-treated polydimethylsiloxane surfaces as enhanced triboelectric nanogenerators," Nano Energy, vol. 15, pp. 523-529, 2015.

[20] Y. Su, J. Chen, Z. Wu, and Y. Jiang, "Low temperature dependence of triboelectric effect for energy harvesting and selfpowered active sensing," Applied Physics Letters, vol. 106, no. 1, Article ID 013114, 2015.

[21] Y. Yang, H. Zhang, Z.-H. Lin et al., "Human skin based triboelectric nanogenerators for harvesting biomechanical energy and as self-powered active tactile sensor system," ACS Nano, vol. 7, no. 10, pp. 9213-9222, 2013.

[22] W. Seung, M. K. Gupta, K. Y. Lee et al., "Nanopatterned textilebased wearable triboelectric nanogenerator," ACS Nano, vol. 9, no. 4, pp. 3501-3509, 2015.

[23] P. Bai, G. Zhu, Y. Liu et al., "Cylindrical rotating triboelectric nanogenerator," ACS Nano, vol. 7, no. 7, pp. 6361-6366, 2013.

[24] G. Zhu, J. Chen, T. Zhang, Q. Jing, and Z. L. Wang, "Radialarrayed rotary electrification for high performance triboelectric generator," Nature Communications, vol. 5, article 3426, 2014.

[25] Y. Yang, G. Zhu, H. Zhang et al., "Triboelectric nanogenerator for harvesting wind energy and as self-powered wind vector sensor system," ACS Nano, vol. 7, no. 10, pp. 9461-9468, 2013.

[26] Z. Wen, J. Chen, M. Yeh et al., "Blow-driven triboelectric nanogenerator as an active alcohol breath analyzer," Nano Energy, vol. 16, pp. 38-46, 2015.

[27] Z.-H. Lin, G. Cheng, W. Wu, K. C. Pradel, and Z. L. Wang, "Dual-mode triboelectric nanogenerator for harvesting water energy and as a self-powered ethanol nanosensor," ACS Nano, vol. 8, no. 6, pp. 6440-6448, 2014.

[28] S. Jung, J. Lee, T. Hyeon, M. Lee, and D.-H. Kim, "Fabricbased integrated energy devices for wearable activity monitors," Advanced Materials, vol. 26, no. 36, pp. 6329-6334, 2014.

[29] H. Zhang, Y. Yang, Y. Su et al., “Triboelectric nanogenerator as self-powered active sensors for detecting liquid/gaseous water/ ethanol," Nano Energy, vol. 2, no. 5, pp. 693-701, 2013.

[30] Y. Su, G. Zhu, W. Yang et al., "Triboelectric sensor for selfpowered tracking of object motion inside tubing," ACS Nano, vol. 8, no. 4, pp. 3843-3850, 2014.

[31] G. Zhu, W. Q. Yang, T. Zhang et al., "Self-powered, ultrasensitive, flexible tactile sensors based on contact electrification," Nano Letters, vol. 14, no. 6, pp. 3208-3213, 2014.

[32] F. Yi, L. Lin, S. Niu et al., "Stretchable-rubber-based triboelectric nanogenerator and its application as self-powered body motion sensors," Advanced Functional Materials, vol. 25, no. 24, pp. 3688-3696, 2015.
[33] Y. Wu, Q. Jing, J. Chen et al., "A self-powered angle measurement sensor based on triboelectric nanogenerator," Advanced Functional Materials, vol. 25, no. 14, pp. 2166-2174, 2015.

[34] P. Bai, G. Zhu, Q. Jing et al., “Transparent and flexible barcode based on sliding electrification for self-powered identification systems," Nano Energy, vol. 12, pp. 278-286, 2015.

[35] Z. L. Wang, J. Chen, and L. Lin, "Progress in triboelectric nanogenerators as a new energy technology and self-powered sensors," Energy \& Environmental Science, vol. 8, no. 8, pp. 22502282, 2015.

[36] G. Zhu, B. Peng, J. Chen, Q. Jing, and Z. L. Wang, “Triboelectric nanogenerators as a new energy technology: from fundamentals, devices, to applications," Nano Energy, vol. 14, pp. 126-138, 2015.

[37] S. Park, H. Kim, M. Vosgueritchian et al., "Stretchable energyharvesting tactile electronic skin capable of differentiating multiple mechanical stimuli modes," Advanced Materials, vol. 26, no. 43, pp. 7324-7332, 2014.

[38] F.-R. Fan, L. Lin, G. Zhu, W. Wu, R. Zhang, and Z. L. Wang, "Transparent triboelectric nanogenerators and self-powered pressure sensors based on micropatterned plastic films," Nano Letters, vol. 12, no. 6, pp. 3109-3114, 2012.

[39] J. Yang, J. Chen, Y. Su et al., "Eardrum-inspired active sensors for self-powered cardiovascular system characterization and throat-attached anti-interference voice recognition," Advanced Materials, vol. 27, no. 8, pp. 1316-1326, 2015.

[40] S. Lee, W. Ko, Y. Oh et al., "Triboelectric energy harvester based on wearable textile platforms employing various surface morphologies," Nano Energy, vol. 12, pp. 410-418, 2015.

[41] G. Zhu, J. Chen, Y. Liu et al., "Linear-grating triboelectric generator based on sliding electrification," Nano Letters, vol. 13, no. 5, pp. 2282-2289, 2013.

[42] S. Wang, L. Lin, Y. Xie, Q. Jing, S. Niu, and Z. L. Wang, "Sliding-triboelectric nanogenerators based on in-plane chargeseparation mechanism," Nano Letters, vol. 13, no. 5, pp. 22262233, 2013.

[43] S. Niu, Y. Liu, S. Wang et al., "Theoretical investigation and structural optimization of single-electrode triboelectric nanogenerators," Advanced Functional Materials, vol. 24, no. 22, pp. 3332-3340, 2014.

[44] Y. Li, G. Cheng, Z.-H. Lin, J. Yang, L. Lin, and Z. L. Wang, "Single-electrode-based rotationary triboelectric nanogenerator and its applications as self-powered contact area and eccentric angle sensors," Nano Energy, vol. 11, pp. 323-332, 2015.

[45] B. Meng, W. Tang, Z.-H. Too et al., "A transparent singlefriction-surface triboelectric generator and self-powered touch sensor," Energy and Environmental Science, vol. 6, no. 11, pp. 3235-3240, 2013.

[46] Y. Yang, H. Zhang, J. Chen et al., "Single-electrode-based sliding triboelectric nanogenerator for self-powered displacement vector sensor system," ACS Nano, vol. 7, no. 8, pp. 7342-7351, 2013.

[47] S. Wang, Y. Xie, S. Niu, L. Lin, and Z. L. Wang, "Freestanding triboelectric-layer-based nanogenerators for harvesting energy from a moving object or human motion in contact and noncontact modes," Advanced Materials, vol. 26, no. 18, pp. 28182824, 2014.

[48] H. Guo, J. Chen, M. H. Yeh et al., "An ultra-robust high-performance triboelectric nanogenerator based on charge replenishment," ACS Nano, vol. 9, no. 5, pp. 5577-5584, 2015. 
[49] L. Lin, S. Wang, S. Niu, C. Liu, Y. Xie, and Z. L. Wang, "Noncontact free-rotating disk triboelectric nanogenerator as a sustainable energy harvester and self-powered mechanical sensor," ACS Applied Materials and Interfaces, vol. 6, no. 4, pp. 30313038, 2014.

[50] H. Guo, Q. Leng, X. He et al., "A triboelectric generator based on checker-like interdigital electrodes with a sandwiched PET thin film for harvesting sliding energy in all directions," Advanced Energy Materials, vol. 5, no. 1, Article ID 1400790, pp. 3031-3038, 2015.

[51] Z. Lin, G. Cheng, X. Li, P. Yang, X. Wen, and Z. Lin Wang, "A multi-layered interdigitative-electrodes-based triboelectric nanogenerator for harvesting hydropower," Nano Energy, vol. 15, pp. 256-265, 2015.

[52] T. Jiang, X. Chen, C. B. Han, W. Tang, and Z. L. Wang, "Theoretical study of rotary freestanding triboelectric nanogenerators," Advanced Functional Materials, vol. 25, no. 19, pp. 2928-2938, 2015.

[53] W. Yang, J. Chen, Q. Jing et al., "3D stack integrated triboelectric nanogenerator for harvesting vibration energy," Advanced Functional Materials, vol. 24, no. 26, pp. 4090-4096, 2014.

[54] J. Chen, G. Zhu, W. Yang et al., "Harmonic-resonator-based triboelectric nanogenerator as a sustainable power source and a self-powered active vibration sensor," Advanced Materials, vol. 25, no. 42, pp. 6094-6099, 2013.

[55] W. Du, X. Han, L. Lin et al., "A three dimensional multilayered sliding triboelectric nanogenerator," Advanced Energy Materials, vol. 4, no. 11, Article ID 1301592, 2014.

[56] W. Yang, J. Chen, G. Zhu et al., "Harvesting energy from the natural vibration of human walking," ACS Nano, vol. 7, no. 12, pp. 11317-11324, 2013.

[57] Y. Xie, S. Wang, S. Niu et al., "Grating-structured freestanding triboelectric-layer nanogenerator for harvesting mechanical energy at 85\% total conversion efficiency," Advanced Materials, vol. 26, no. 38, pp. 6599-6607, 2014.

[58] W. Tang, T. Jiang, F. R. Fan et al., "Liquid-metal electrode for high-performance triboelectric nanogenerator at an instantaneous energy conversion efficiency of 70.6\%," Advanced Functional Materials, vol. 25, no. 24, pp. 3718-3725, 2015.

[59] G. Zhu, Y. S. Zhou, P. Bai et al., "A shape-adaptive thin-filmbased approach for 50\% high-efficiency energy generation through micro-grating sliding electrification," Advanced Materials, vol. 26, no. 23, pp. 3788-3796, 2014.

[60] L. Lin, Y. Xie, S. Niu, S. Wang, P.-K. Yang, and Z. L. Wang, "Robust triboelectric nanogenerator based on rolling electrification and electrostatic induction at an instantaneous energy conversion efficiency of 55\%," ACS Nano, vol. 9, no. 1, pp. 922930, 2015.

[61] J. Chen, J. Yang, H. Guo et al., "Automatic mode transition enabled robust triboelectric nanogenerators," ACS Nano, vol. 9, no. 12, pp. 12334-12343, 2015.

[62] L. Zhang, B. Zhang, J. Chen et al., "Lawn structured triboelectric nanogenerators for scavenging sweeping wind energy on rooftops," Advanced Materials, vol. 28, no. 8, pp. 1650-1656, 2016.

[63] Y. Wu, X. Zhong, X. Wang, Y. Yang, and Z. L. Wang, "Hybrid energy cell for simultaneously harvesting wind, solar, and chemical energies," Nano Research, vol. 7, no. 11, pp. 1631-1639, 2014.

[64] E. Briones, J. Briones, A. Cuadrado et al., "Seebeck nanoantennas for solar energy harvesting," Applied Physics Letters, vol. 105, no. 9, Article ID 093108, 2014.
[65] L. Zheng, Z.-H. Lin, G. Cheng et al., "Silicon-based hybrid cell for harvesting solar energy and raindrop electrostatic energy," Nano Energy, vol. 9, pp. 291-300, 2014.

[66] Y. Hu, J. Yang, S. Niu, W. Wu, and Z. L. Wang, "Hybridizing triboelectrification and electromagnetic induction effects for high-efficient mechanical energy harvesting," ACS Nano, vol. 8, no. 7, pp. 7442-7450, 2014.

[67] Y. Yang, H. Zhang, J. Chen, S. Lee, T.-C. Hou, and Z. L. Wang, "Simultaneously harvesting mechanical and chemical energies by a hybrid cell for self-powered biosensors and personal electronics," Energy \& Environmental Science, vol. 6, no. 6, pp. 1744-1749, 2013.

[68] Y. Zi, L. Lin, J. Wang et al., "Triboelectric-pyroelectric-piezoelectric hybrid cell for high-efficient energy-harvesting and self-powered sensing," Advanced Materials, vol. 27, no. 14, pp. 2340-2347, 2015.

[69] L. Zheng, G. Cheng, J. Chen et al., "A hybridized power panel to simultaneously generate electricity from sunlight, raindrops, and wind around the clock," Advanced Energy Materials, vol. 5, no. 21, 2015.

[70] L. Zheng, G. Cheng, J. Chen et al., "A hybridized power panel to simultaneously generate electricity from sunlight, raindrops and wind around the clock," Advanced Energy Materials, vol. 5, no. $4,2015$.

[71] Y. Yang, H. Zhang, Z.-H. Lin et al., "A hybrid energy cell for selfpowered water splitting," Energy \& Environmental Science, vol. 6, no. 8, pp. 2429-2434, 2013.

[72] Y. Yang, H. Zhang, Y. Liu et al., "Silicon-based hybrid energy cell for self-powered electrodegradation and personal electronics," ACS Nano, vol. 7, no. 3, pp. 2808-2813, 2013.

[73] J. Chen, G. Zhu, J. Yang et al., "Personalized keystroke dynamics for self-powered human-machine interfacing," ACS Nano, vol. 9, no. 1, pp. 105-116, 2015.

[74] W. Yang, J. Chen, X. Wen et al., "Triboelectrification based motion sensor for human-machine interfacing," ACS Applied Materials \& Interfaces, vol. 6, no. 10, pp. 7479-7484, 2014.

[75] S. Y. Kuang, J. Chen, X. B. Cheng, G. Zhu, and Z. L. Wang, “Twodimensional rotary triboelectric nanogenerator as a portable and wearable power source for electronics," Nano Energy, vol. 17, pp. 10-16, 2015.

[76] G. Zhu, P. Bai, J. Chen, and Z. Lin Wang, "Power-generating shoe insole based on triboelectric nanogenerators for selfpowered consumer electronics," Nano Energy, vol. 2, no. 5, pp. 688-692, 2013.

[77] T.-C. Hou, Y. Yang, H. Zhang, J. Chen, L.-J. Chen, and Z. Lin Wang, "Triboelectric nanogenerator built inside shoe insole for harvesting walking energy," Nano Energy, vol. 2, no. 5, pp. 856$862,2013$.

[78] N. Zhang, J. Chen, Y. Huang et al., "A wearable all-solid photovoltaic textile," Advanced Materials, vol. 28, no. 2, pp. 263-269, 2016.

[79] P. Bai, G. Zhu, Z.-H. Lin et al., "Integrated multilayered triboelectric nanogenerator for harvesting biomechanical energy from human motions," ACS Nano, vol. 7, no. 4, pp. 3713-3719, 2013.

[80] J. Yang, J. Chen, Y. Liu, W. Yang, Y. Su, and Z. L. Wang, "Triboelectrification-based organic film nanogenerator for acoustic energy harvesting and self-powered active acoustic sensing," ACS Nano, vol. 8, no. 3, pp. 2649-2657, 2014.

[81] X. Fan, J. Chen, J. Yang, P. Bai, Z. Li, and Z. L. Wang, "Ultrathin, rollable, paper-based triboelectric nanogenerator for acoustic 
energy harvesting and self-powered sound recording," ACS Nano, vol. 9, no. 4, pp. 4236-4243, 2015.

[82] Z. Li, J. Chen, J. Yang et al., “ $\beta$-cyclodextrin enhanced triboelectrification for self-powered phenol detection and electrochemical degradation," Energy \& Environmental Science, vol. 8, no. 3, pp. 887-896, 2015.

[83] Z. Li, J. Chen, H. Guo et al., "Triboelectrification-enabled selfpowered detection and removal of heavy metal ions in wastewater," Advanced Materials, 2016.

[84] P. Bai, G. Zhu, Q. Jing et al., "Membrane-based self-powered triboelectric sensors for pressure change detection and its uses in security surveillance and healthcare monitoring," Advanced Functional Materials, vol. 24, no. 37, pp. 5807-5813, 2014.

[85] G. Zhu, Y. Su, P. Bai et al., "Harvesting water wave energy by asymmetric screening of electrostatic charges on nanostructured hydrophobic thin-film surfaces," ACS Nano, vol. 8, no. 6, pp. 10424-10432, 2014.

[86] Y. Su, X. Wen, G. Zhu et al., "Hybrid triboelectric nanogenerator for harvesting water wave energy and as a self-powered distress signal emitter," Nano Energy, vol. 9, pp. 186-195, 2014.

[87] Y. Hu, J. Yang, Q. Jing, S. Niu, W. Wu, and Z. L. Wang, “Triboelectric nanogenerator built on suspended 3D spiral structure as vibration and positioning sensor and wave energy harvester," ACS Nano, vol. 7, no. 11, pp. 10424-10432, 2015.

[88] Q. Jing, G. Zhu, P. Bai et al., "Case-encapsulated triboelectric nanogenerator for harvesting energy from reciprocating sliding motion," ACS Nano, vol. 8, no. 4, pp. 3836-3842, 2014.

[89] Z.-H. Lin, G. Zhu, Y. S. Zhou et al., "A self-powered triboelectric nanosensor for mercury ion detection," Angewandte ChemieInternational Edition, vol. 52, no. 19, pp. 5065-5069, 2013.

[90] W. Yang, Z. Liu, J. Chen et al., "A high-performance whitelight-emitting-diodes based on nano-single crystal divanadates quantum dots," Scientific Reports, vol. 5, Article ID 10460, 2015.

[91] J. Chen, J. Yang, Z. Li et al., "Networks of triboelectric nanogenerators for harvesting water wave energy: a potential approach toward blue energy," ACS Nano, vol. 9, no. 3, pp. 3324-3331, 2015. 

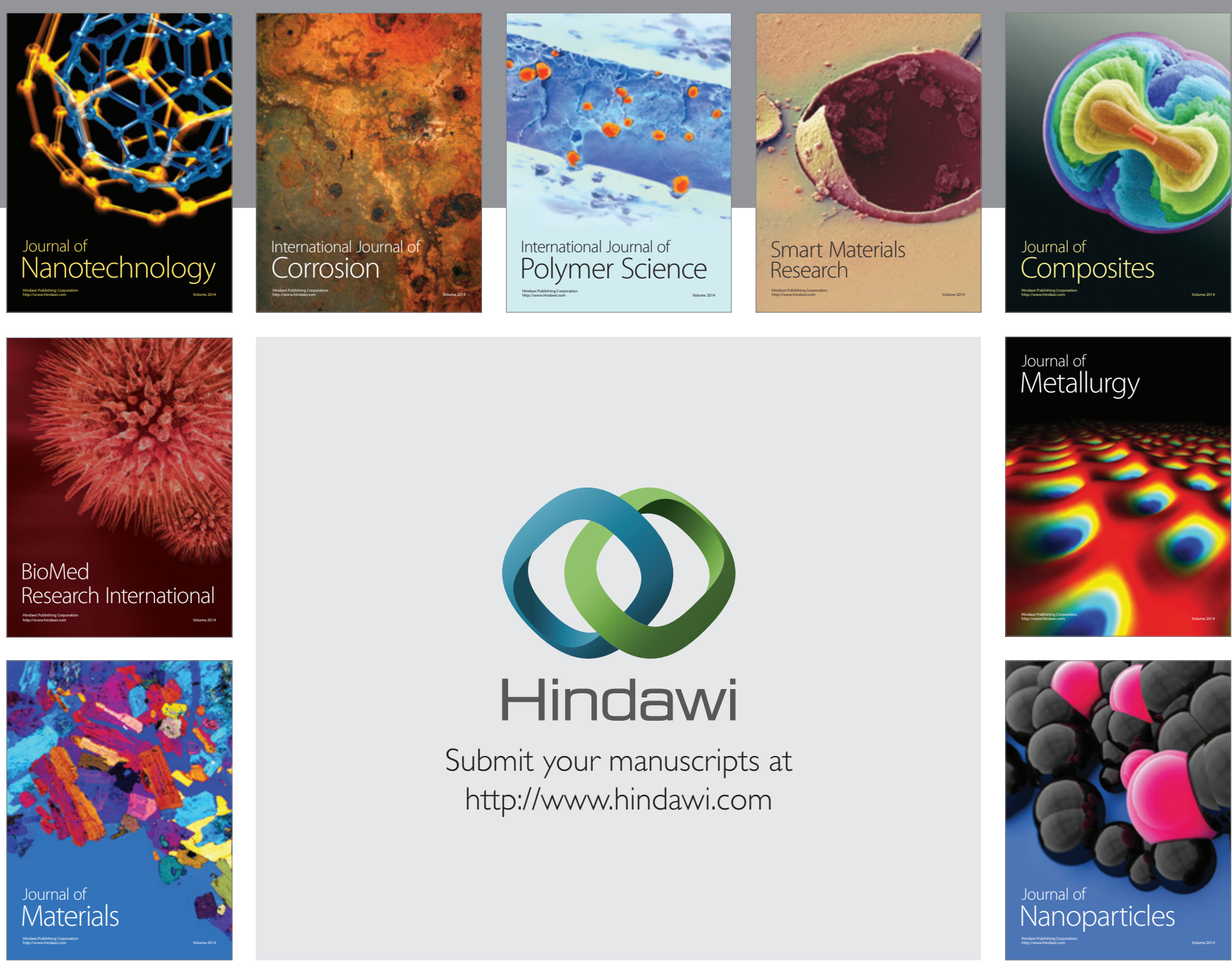

\section{Hindawi}

Submit your manuscripts at

http://www.hindawi.com

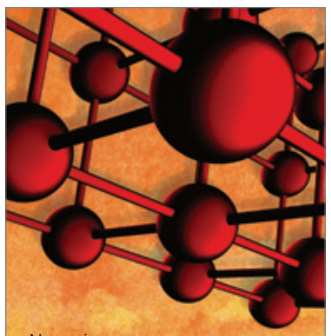

Materials Science and Engineering
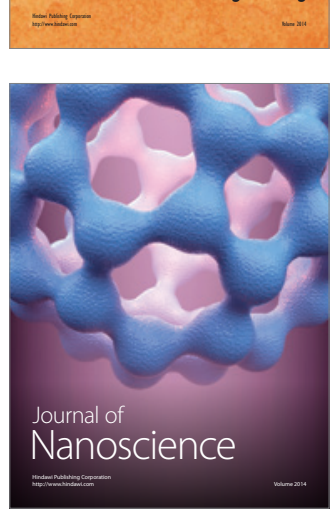
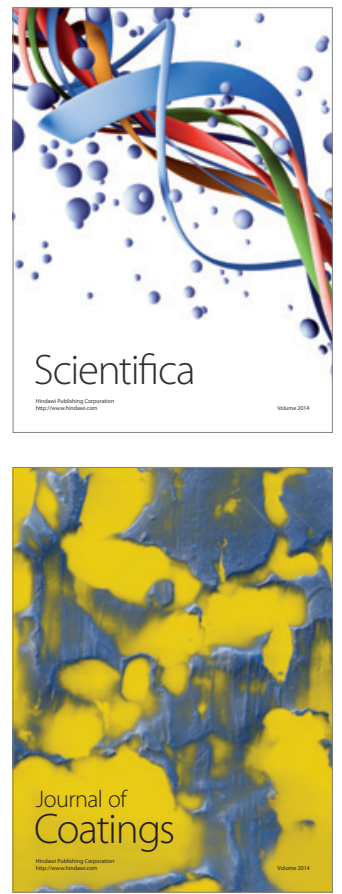
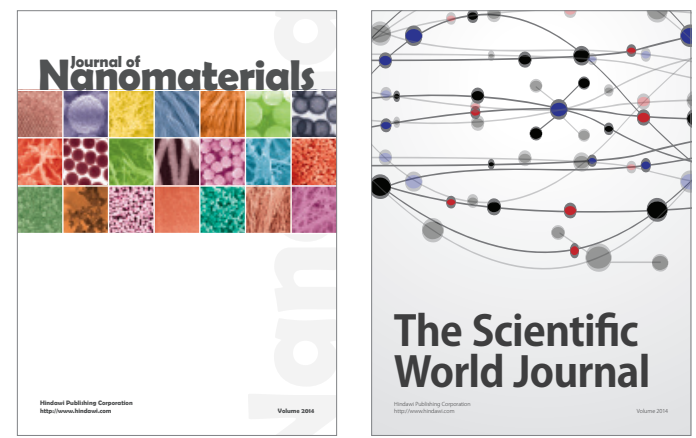

The Scientific World Journal
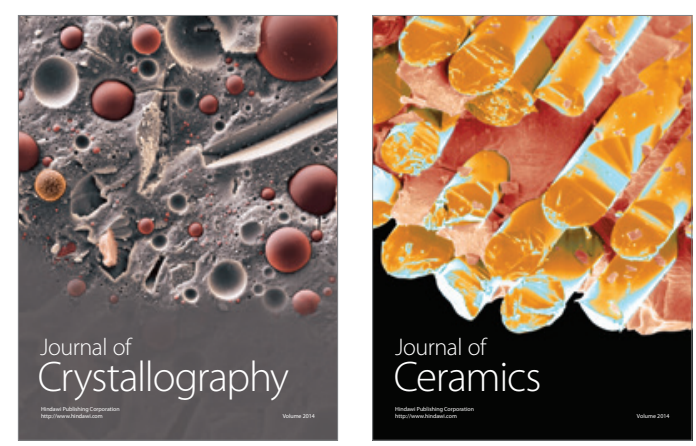
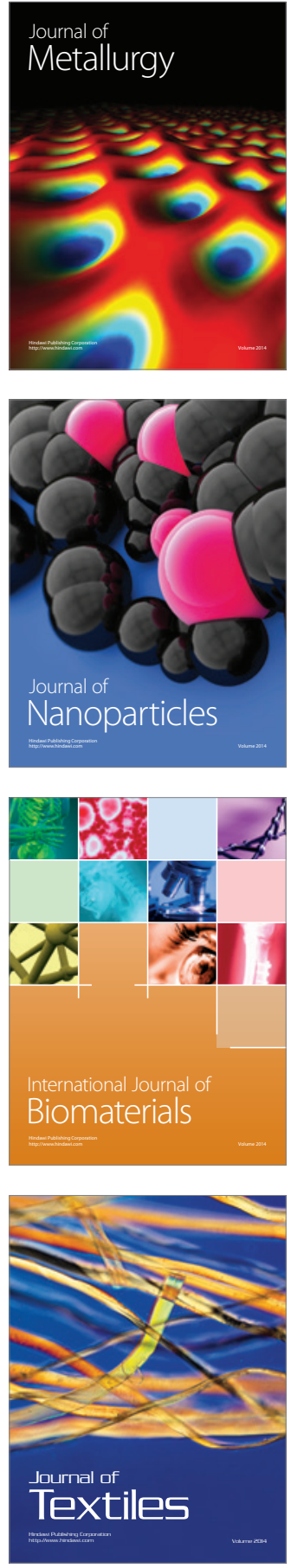